Trinity University

Digital Commons @ Trinity

Mathematics Faculty Research

Mathematics Department

2017

\title{
Global Stability of Higher Dimensional Monotone Maps
}

Eduardo C. Balreira

Trinity University, ebalreir@trinity.edu

Saber Elaydi

Trinity University, selaydi@trinity.edu

Rafael Luís

Follow this and additional works at: https://digitalcommons.trinity.edu/math_faculty

Part of the Mathematics Commons

\section{Repository Citation}

Balreira, E. C., Elaydi, S. \& Luís, R. (2017). Global stability of higher dimensional monotone maps. Journal of Difference Equations and Applications, 23(12), 2037-2071. http://doi.org/10.1080/ 10236198.2017.1388375

This Article is brought to you for free and open access by the Mathematics Department at Digital Commons @ Trinity. It has been accepted for inclusion in Mathematics Faculty Research by an authorized administrator of Digital Commons @ Trinity. For more information, please contact jcostanz@trinity.edu. 


\title{
Global stability of higher dimensional monotone maps
}

\author{
E. Cabral Balreira ${ }^{\mathrm{a}}$, Saber Elaydi ${ }^{\mathrm{a}}$ and Rafael Luís ${ }^{\mathrm{b}}$ \\ ${ }^{a}$ Department of Mathematics, Trinity University, San Antonio, TX, USA; ${ }^{b}$ Center for Mathematical Analysis, \\ Geometry, and Dynamical Systems, Instituto Superior Técnico, University of Lisbon, Lisbon, Portugal
}

\begin{abstract}
We develop a notion of monotonicity for maps defined on Euclidean spaces $\mathbb{R}_{+}^{k}$, of arbitrary dimension $k$. This is a geometric approach that extends the classical notion of planar monotone maps or planar competitive difference equations. For planar maps, we show that our notion and the classical notion of monotonicity are equivalent. In higher dimensions, we establish certain verifiable conditions under which Kolmogorov monotone maps on $\mathbb{R}_{+}^{k}$ have a globally asymptotically stable fixed point. We apply our results to two competition population models, the Leslie-Gower and the Ricker models of two- and three-species. It is shown that these two models have a unique interior fixed point that is globally asymptotically stable.
\end{abstract}

\section{ARTICLE HISTORY}

Received 26 April 2017

Accepted 28 September

2017

\section{KEYWORDS}

Global stability; monotone maps; Ricker competition model; Leslie-Gower competition model; competitive maps

\section{AMS SUBJECT} CLASSIFICATIONS

37C75; 39A30; 37N25; 39A60

\section{Introduction}

One of the fundamental problems in the area of discrete dynamical systems is the global stability of periodic or fixed points. In this paper we shall consider continuous maps $F: \mathbb{R}_{+}^{k} \rightarrow \mathbb{R}_{+}^{k}$, where $\mathbb{R}_{+}^{k}=[0, \infty)^{k}$. The map $F$ generates a discrete dynamical system or a difference equation of the form

$$
\mathbf{x}_{n+1}=F\left(\mathbf{x}_{n}\right),
$$

where $F^{n}\left(\mathbf{x}_{0}\right)=\mathbf{x}_{n}$ and $F^{n}$ is the $n$th composition of the map $F$. A fixed point $\mathbf{x}^{*}$ of the map $F$ is said to be globally asymptotically stable if it is stable and globally attracting in the sense that its basin of attraction is the entire interior of $\mathbb{R}_{+}^{k}$, see $[12,28]$ for more details.

The first comprehensive investigation of global stability of maps was initiated by LaSalle [23]. His approach is to construct a Liapunov function which would show whether or not a fixed point of a difference equation is globally or locally asymptotically stable. Due to the lack of a systematic method to construct suitable Liapunov functions, this approach has had a limited success $[11,20]$. Focusing on planar maps, the authors [6] used singularity theory due to Whitney [33] to show that, under certain conditions, local stability implies global stability. Extending this approach to higher-dimensional maps is yet to be done as singularities are not classified as well as in the plane.

More successful efforts have been directed towards a special class of maps. One such special class is the class of triangular maps [5,9]. These are maps for which the Jacobian matrix is upper or lower triangular. Here one can show that, under certain conditions, every 
orbit in the domain converges to a fixed point and, consequently, if one has a unique fixed point then it must be globally asymptotically stable. In applications to biology, triangular maps model competition systems with hierarchy [2].

In this paper, our focus will be on another special class of maps, monotone maps. Hal Smith [32] has established an effective method to show global stability for monotone planar maps.

Unfortunately, extending Smith's results to maps on $\mathbb{R}_{+}^{k}$ has alluded many researchers for several decades. On a more positive note, several authors have succeeded in proving the existence of a global attractor, called the carrying simplex, for a certain class of maps, for instance, Herrera [16], Hirsch [18], Wang and Jiang [21]. A carrying simplex for the map $F$ is a compact invariant hypersurface $\Sigma \subset \mathbb{R}_{+}^{k}$ such that the omega limit set of every orbit (except the origin) lies in $\Sigma$. It should be noted that Hirsch was the first to prove, under certain conditions on a map, the existence, uniqueness, and the global attractivity of the carrying simplex [18, Theorem 1$]$.

In this paper, we assume that our maps possess the carrying simplex. For this purpose, we are going to use the results given by Herrera [16, Corollary 6.1] which give conditions under which a map possesses a unique carrying simplex. Our main aim, however, is to establish a general theory of the global stability of the unique fixed point of monotone maps or competitive systems on $\mathbb{R}_{+}^{k}$. We accomplish our task by developing a geometric notion of monotonicity that does not depend on the notion of competitiveness. Roughly speaking, a map is normally monotone if it preserves positive normals (a more precise definition will be given in the next section). It should be noted that for planar maps, our notion of monotonicity coincides with that of Smith. Henceforth, we will omit the prefix normally when we talk about monotone maps.

The last section of the paper is dedicated to demonstrate the effectiveness of our results using concrete examples. We provide detailed proofs of the global stability of two important competition models, the planar and the three-dimensional Leslie-Gower competition model [25] and the Ricker competition model [29]. This illustrates how our theory can be applied beyond the theory of monotone planar maps of Smith [32] and what are the techniques required to verify our set of conditions in applications.

\section{Geometric monotonicity and main results}

Given a map $F: \mathbb{R}^{k} \rightarrow \mathbb{R}^{k}$, we say $F$ is order preserving if it preserves an order generated by an orthant. Formally, each of the orthants $\mathcal{O}$ of $\mathbb{R}^{k}$ generates a partial order $\leq \mathcal{O}$ where $\mathbf{x} \leq \mathcal{O} \mathbf{y}$ if and only if $\mathbf{y}-\mathbf{x} \in \mathcal{O}$. We also write $\mathbf{x}<\mathcal{O}$ y when $\mathbf{y}-\mathbf{x} \in \mathcal{O} \backslash\{0\}$. In the case when $\mathcal{O}$ is the positive orthant $\mathbb{R}_{+}^{k}$, we denote the induced order as the canonical order and simply drop the subscript above. In addition, it may be useful to consider an orthant generated by a vector $\mathbf{v}$, that is, all vectors that belong to the same orthant as v. Formally, we can define the orthant generated by $\mathbf{v}$ to be denoted by $\mathcal{O}(\mathbf{v})=\left\{\mathbf{x} \in \mathbb{R}^{k} \mid \operatorname{sgn}\left(x_{i}\right)=\operatorname{sgn}\left(v_{i}\right)\right\}$.

We are also interested with the restriction of a map and the order relation within to a subspace of $\mathbb{R}^{k}$. The following definition makes it easier to handle these situations. Indeed, for $I=\left\{i_{1}, i_{2}, \ldots, i_{n}\right\} \subseteq\{1, \ldots, k\}$, let $H_{I}$ be the subspace spanned by the coordinate axes in $I$. Formally, we consider the coordinate axes to be generated by the canonical vectors $\left\{\mathbf{e}_{1}, \mathbf{e}_{2}, \ldots, \mathbf{e}_{k}\right\}$, where each $\mathbf{e}_{i}$ is a vector with all coordinates zero but one in coordinate $i$. Then we let $H_{I}=\mathbb{R}^{k} \cap \operatorname{span}\left\{\mathbf{e}_{i_{1}}, \mathbf{e}_{i_{2}}, \ldots, \mathbf{e}_{i_{n}}\right\}$ and for a set $\Omega \subseteq \mathbb{R}^{k}$, we denote $\Omega_{I}=H_{I} \cap \Omega$. 
Next, we also consider the projections onto such subspaces, i.e. consider $\pi_{I}: \mathbb{R}^{k} \rightarrow H_{I}$ to be the projection of a point in $\mathbb{R}^{k}$ into its coordinates in $H_{I}$. Finally, in an effort to ease the notation, we hope that it will be clear from context when $H_{I}$ refers to the positive cone instead of using $H_{I}^{+}$.

With this notation in place, we can say that a map $F: \Omega \rightarrow \mathbb{R}^{k}$ is $\mathcal{O}$-order preserving if whenever $\mathbf{x} \leq \mathcal{O} \mathbf{y}$, then $F(\mathbf{x}) \leq \mathcal{O} F(\mathbf{y})$. It is common to say that a map is monotone if it is order preserving for at least one orthant. In the study of monotone planar maps, one has essentially two orderings induced by the first or third quadrant and the second or fourth quadrant. In the work of Smale [30] and Smith [32], these maps are defined as cooperative and competitive maps. Later in a seminal paper, Smith [31] initiated the theory of higher dimensional monotone maps, but the examples given and recent developments in the field have been done on planar or essentially planar maps.

Our goal is to develop a geometric theory of monotone maps. We provide a new definition of monotonicity of maps that uses the notion of preservation of normals of hypersurfaces that can yield results on global stability in higher dimensions. Our definition will be shown to be equivalent to the usual definition of competitive planar maps.

Let us recall the idea of a normal vector and refer the reader to [24] for complete details on basic concepts from differential geometry. Given an oriented differentiable hypersurface $\Gamma \subseteq \mathbb{R}^{k}$ and $\mathbf{p} \in \Gamma$, let $\eta_{\Gamma}(\mathbf{p})$ denote the normal vector to the tangent space $T_{\Gamma}(\mathbf{p})$ at $\mathbf{p}$.

Definition 2.1: An open set $R \subseteq \mathbb{R}_{+}^{k}$ is a monotone region if $\Gamma=\partial R$ is a hypersurface such that for any $\mathbf{p} \in \Gamma \cap \operatorname{int}\left(\mathbb{R}_{+}^{k}\right)$, we have $\eta_{\Gamma}(\mathbf{p})>0$. That is, the normal at every point in the boundary of $\Omega$ not in a coordinate subspace is a positive vector.

Intuitively, a monotone region is a region bounded by the positive cone and a hypersurface with positive normal. Observe that if $R=\mathbb{R}_{+}^{k}$, vacuously $\mathbb{R}_{+}^{k}$ is a monotone region. We are now ready to give our definition of monotonicity for maps.

Definition 2.2: $\quad$ Let $F: \Omega \rightarrow \mathbb{R}_{+}^{k}$ be a local diffeomorphism of class $C^{1}$. We say that $F$ is monotone at $\mathbf{p}$ if for any hypersurface $\Gamma$ containing $\mathbf{p}$ with $\eta_{\Gamma}(\mathbf{p})>0$, we have $\eta_{F(\Gamma)}(F(\mathbf{p}))>0$. We say $F$ monotone if it is so at every point.

Simply said, a map $F$ is monotone if it preserves positive normals. Viewed geometrically, a monotone map has the property that it preserves the structure of graphs. In fact, if $\Gamma$ satisfies $\eta_{\Gamma}(\mathbf{p})>0$, then by the implicit function theorem the hypersurface $\Gamma$ can be viewed as a graph of a function with respect to any subspace $H_{I}$. This is consistent with the usual definition of monotone maps in one dimension. Indeed, the image of an interval can be viewed as the graph of a function with respect to both axes. We will show later that this is also the case for monotone maps in two dimensions.

In general, if one is only concerned with the graph preservation property, then the work of Basu, Gabrielov, and Vorobjov [7,8] establishes an important theory on general monotone sets. From our perspective, as we are interested in global stability, we will focus on the dynamics of monotone maps and how we can analytically verify whether a map is monotone in $\mathbb{R}_{+}^{k}$. In fact, to check if an orientation preserving map is normally monotone, it suffices to check that the inverse Jacobian matrix is a positive matrix, that is, entries of $J F^{-1}(\mathbf{p})$ are positive. This is similar to the original analytic condition proposed by Smith [31] and it will be verified by a straightforward computation using the Cauchy-Binet formula [22] to find the determinant of the product of matrices. 
Another concept we will use in our geometric theory of monotone maps is the idea of a carrying simplex $\Sigma$, informally this is an attracting hypersurface of the dynamics of our system. This has been well-study by Baigent [3], Hirsch [18], and Herrera [16] and here we provide the formal definition.

Definition 2.3: We say that $F: \mathbb{R}_{+}^{k} \rightarrow \mathbb{R}_{+}^{k}$ admits a carrying simplex, denoted by $\Sigma \subseteq \mathbb{R}_{+}^{k} \backslash\{0\}$, if the following hold.

(i) $\Sigma$ is homeomorphic to a $(n-1)$-simplex.

(ii) $\quad \Sigma$ is unordered, that is, if $\mathbf{x}, \mathbf{y} \in \Sigma$ and $\mathbf{y} \geq \mathbf{x}$ then $\mathbf{y}=\mathbf{x}$.

(iii) $\quad \Sigma$ is invariant under $F$ and $F: \Sigma \rightarrow \Sigma$ is a homeomorphism.

(iv) For every $\mathbf{x}>0$, there exists $\mathbf{y} \in \Sigma$ such that $\lim _{n \rightarrow \infty}\left|F^{n}(\mathbf{x})-F^{n}(\mathbf{y})\right|=0$.

As we begin focusing the analyses to global stability of maps, we will make additional assumptions for our maps. Since we are concerned with applications to mathematical biology and mathematical economics, we will restrict ourselves to the case where $F$ is a map of Kolmogorov type, i.e. the map $F$ is given by

$$
F(\mathbf{x})=\left(x_{1} f_{1}(\mathbf{x}), x_{2} f_{2}(\mathbf{x}), \ldots, x_{k} f_{k}(\mathbf{x})\right),
$$

where $f_{i}: \mathbb{R}_{+}^{k} \rightarrow \mathbb{R}_{+}$and $\mathbf{x}=\left(x_{1}, x_{2}, \ldots, x_{k}\right)$. The main feature of this class of maps is that the origin is a fixed point and all the coordinate hyperplanes are invariant. The hypotheses that are to follow are similar to those made by Smale, Smith, Hsu, and Waltman [19,30-32].

(H1) There exists a monotone region $R$ such that $\left.F\right|_{R}$ is orientation preserving local homeomorphism with $F(\Omega \backslash R) \subseteq F(R)$.

(H2) The map $\left.F\right|_{R}$ is a monotone map.

(H3) For each $I \subseteq\{1,2 \ldots, k\}$ with $|I|=k-1$, the restriction map $\left.F\right|_{\Omega_{I}}$ is invariant in $\Omega_{I}$ and $\left.F\right|_{\Omega_{I}}$ has a unique interior fixed point $E_{I}$ that is globally asymptotically stable in $\Omega_{I}$, but a saddle in $\Omega$.

(H4) The map $F$ admits a carrying simplex.

In order to verify (H1), one must find a monotone region $R$ that has the property that $F(R)$ does not contain any critical points and for any $\mathbf{x} \in \Omega$, either $\mathbf{x} \in R$ or $F(\mathbf{x}) \in F(R)$. For instance, this can be done when the region bounded by the set of critical points is the monotone region $R$ and the image of $F$ is contained in $R$. For conditions (H2) and (H3), we will show that one must simply check analytic conditions. Indeed, we shall verify that the Jacobian matrix of $F$ has all the information required to check (H2) and standard methods of stability of fixed points are used to check (H3). Next, to check (H4), the work in [17] has established analytic conditions under which $F$ admits a carrying simplex which are checked to be satisfied for the Leslie-Gower and Ricker competition models in higher dimensions and can be extended to other models as well.

We are now ready to state our main result establishing global stability of monotone maps.

Theorem 2.4: Assume that $F: \Omega \rightarrow \Omega$ satisfies (H1)-(H4). If $F$ has a unique positive coexistence fixed point $E^{*}$, then $E^{*}$ is globally asymptotically stable in the interior of $\Omega$.

The proof of Theorem 2.4 is geometric in nature. Due to the interest in planar maps, or competition maps between two species, we first introduce in Section 3 our techniques for two dimensional maps where we will show that our geometric definition of monotone 
planar maps is equivalent to the classical notion of competitive maps. In Section 4, we state and prove our results for higher dimensional maps and, in Section 5, we focus our attention on how to check the hypotheses of our main result for specific competition models.

In addition, we find a sufficient set of conditions that can be directly verified in applications. Let us consider $F: \Omega \rightarrow \Omega$ to be a map of class $C^{1}$ and suppose that the global stability of the model is known in lower dimensions, in particular in dimension $k-1$. More precisely, for each $r=1,2, \ldots, k$, denote $I_{r}=\{1,2, \ldots, k\}-\{r\}$ and assume the map $\left.F\right|_{\Omega_{I_{r}}}$ in invariant in $H_{I_{r}}$ and has a unique coexistence fixed point $E_{r}$ that is globally asymptotically stable.

The following result provides verifiable conditions to check hypotheses (H1)-(H4) and establishing global stability as well.

Corollary 2.5: Let $F: \Omega \rightarrow \Omega$ be a Kolmogorov map as in (2) and satisfying the following conditions:

(a) $\Omega$ is a monotone region.

(b) $\operatorname{det} J F(\mathbf{x})>0$ for any $\mathbf{x} \in \Omega$.

(c) $J F^{-1}(\mathbf{x})>0$ for any $\mathbf{x} \in \Omega$.

(d) For each $r=1,2, \ldots, k$, the eigenvalues of $J F\left(E_{r}\right)$ satisfy $\left|\lambda_{r}\right|>1$ and $\left|\lambda_{i}\right|<1$, for $i \neq r$.

(e) For each $i=1,2, \ldots, k$, we have $f_{i}(\mathbf{y})>f_{i}(\mathbf{x})$ whenever $y_{i} f_{i}(\mathbf{y})>x_{i} f_{i}(\mathbf{x})$.

Then $E^{*}$ is globally asymptotically stable in the interior of $\Omega$.

Note that since $F: \Omega \rightarrow \Omega$, the image is trivially contained in the domain and from (a) and (b) we have that (H1) holds. In Section 4, we will show in Lemma 4.1 that (c) is equivalent to say $F$ is monotone and thus (H2) holds. Next, for (H3), condition (d) is simply the standard methods of stability of fixed points. Finally, it is shown in [17] that condition (e), together with the property that $F$ is retrotone, is needed to establish $(\mathrm{H} 4)$.

Recall that a map $F: \mathbb{R}_{+}^{k} \rightarrow \mathbb{R}_{+}^{k}$ is said to be retrotone if whenever $F(\mathbf{y})>F(\mathbf{x})$, we have $\mathbf{y}>\mathbf{x}$, provided $\mathbf{x}, \mathbf{y} \neq 0$.

We remark that monotonicity, or condition (c), is crucial to show that $F$ is retrotone.

We conclude this section with a discussion of the structure of the stable and unstable manifolds associated with the fixed points and recalling some of the principal properties that we will need. Given an open neighbourhood $U$ of a fixed point $\mathbf{x}^{*}$ of $F$, the local stable manifold for $\mathbf{x}^{*}$ in this neighbourhood is defined to be the set

$$
W_{l o c}^{s}\left(\mathbf{x}^{*}, U\right)=\left\{z \in U \mid F^{n}(z) \in U \text { for } n>0 \text {, and } \lim _{n \rightarrow \infty} F^{n}(z)=\mathbf{x}^{*}\right\}
$$

Before we define the local unstable manifold of $\mathbf{x}^{*}$, let us consider the following notation. Definition 2.6: Let $z \in \mathbb{R}^{k}$. We say that $z$ has a complete negative orbit under $F$, if there exists a sequence denoted by

$$
O^{-}(z)=\left\{z_{-n} \mid z_{0}=z, F\left(z_{-n}\right)=z_{-n+1}, \quad n=1,2,3, \ldots\right\} .
$$

The local unstable manifold of $\mathbf{x}^{*}$ in the neighbourhood $U$ is the set

$$
W_{l o c}^{u}\left(\mathbf{x}^{*}, U\right)=\left\{z \in U \mid \exists O^{-}(z) \subset U \text { such that } \lim _{n \rightarrow \infty} z_{-n}=\mathbf{x}^{*}\right\}
$$




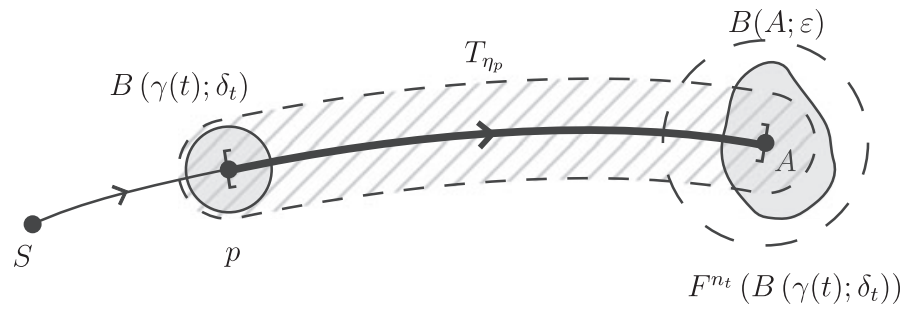

Figure 1. At each point in the interior of the heteroclinic connection, we can find a tubular neighbourhood $T_{\eta_{p}}$ where each point is globally attracted to $A$.

Namely, $W_{l o c}^{u}\left(\mathbf{x}^{*}, U\right)$ is the set of points that have a complete negative orbit converging to $\mathbf{x}^{*}$. The stable manifold theory $[12,28]$ guarantees the existence of the local stable and unstable manifolds in a suitable open neighbourhood $U$ of the fixed point $\mathbf{x}^{*}$. In this case, we denote the stable and unstable manifolds of $\mathbf{x}^{*}$ by $W_{l o c}^{s}\left(\mathbf{x}^{*}\right)$ and $W_{l o c}^{u}\left(\mathbf{x}^{*}\right)$, respectively. Moreover, these manifolds are invariant.

Once we have the local unstable manifold, then the global unstable manifold is given by

$$
W^{u}\left(\mathbf{x}^{*}\right)=\bigcup_{n \geq 0} F^{n}\left(W_{l o c}^{u}\left(\mathbf{x}^{*}\right)\right)
$$

It should be noted that since $F$ is of class $C^{1}$, both $W_{l o c}^{u}$ and $W_{l o c}^{s}$ are $C^{1}$ manifolds with no self-intersection.

Lastly, we will be interested in considering the dynamics of $F$ when restricted to the unstable manifolds. At times, these can be connected in the sense that as we leave the unstable manifold of a fixed point, we may be contained in the stable manifold of another fixed point. Formally, we say the fixed points $\mathbf{x}^{*}$ and $\mathbf{y}^{*}$ are a heteroclinic pair with heteroclinic connection $\gamma$ if $\mathbf{x}^{*}$ and $\mathbf{y}^{*}$ are fixed points of $F$ and $\gamma \subseteq W^{u}\left(\mathbf{x}^{*}\right) \cap W^{s}\left(\mathbf{y}^{*}\right)$.

In the sequel, we need the following general result concerning the dynamics of heteroclinic pairs of a saddle and an attractor.

Lemma 2.7: Let $F: \mathbb{R}_{+}^{k} \rightarrow \mathbb{R}_{+}^{k}$ be a continuous map and $S, A \in \mathbb{R}^{k}$ be a local saddle and local attractor of $F$, respectively. Assume that $S \in \partial \mathbb{R}_{+}^{k}$ with $W^{s}(S) \subseteq \partial \mathbb{R}_{+}^{k}$ and $A \in$ $\operatorname{int}\left(\mathbb{R}_{+}^{k}\right)$. If S and $A$ are a heteroclinic pair with heteroclinic connection $\gamma \subset W^{u}(S) \cap W^{s}(A)$, then there is a neighbourhood $G$ of $\gamma$ such that for all $x \in G \cap \operatorname{int}\left(\mathbb{R}_{+}^{k}\right)$, then $\lim _{n \rightarrow \infty} F^{n}(x)=A$.

Proof: Since $A$ is a local attractor, we know there is $\varepsilon>0$ such that the open ball $B(A ; \varepsilon)$ is contained in the basin of attraction. For every point $p \in \gamma$, except the endpoints, we are able to find a tubular neighbourhood in $\mathbb{R}_{+}^{k}$ starting at $p$ along $\gamma$ up to the local attractor $A$, which we will denote by $T_{\eta_{p}}$. This neighbourhood $T_{\eta_{p}}$ will have the property that orbits that start in $T_{\eta_{p}}$, will eventually be in the basin of attractor of $A$, and thus, will converge to A.

Let us now formalize and provide the details of this idea which is illustrated in Figure 1. Consider a parametrization $\gamma:[0,1] \rightarrow \mathbb{R}_{+}^{k}$ such that $\gamma(0)=S$ and $\gamma(1)=A$. For each $t \in(0,1]$, there is $n_{t} \in \mathbb{N}$ such that for $n \geq n_{t}, F^{n}(\gamma(t)) \in B(A ; \varepsilon)$, since $\gamma \subseteq W^{s}(A)$. By continuity of $F$, and its composition, there is $\delta_{t}>0$ so that $F^{n_{t}}\left(B\left(\gamma(t) ; \delta_{t}\right)\right) \subseteq B(A ; \varepsilon)$. Note that when $t=1$, we can take any $n_{1} \in \mathbb{N}$, as $\gamma(1)=A$ is a fixed point and $\delta_{1}=\varepsilon$. 
More importantly, for any $x \in B\left(\gamma(t) ; \delta_{t}\right)$, the orbits of $x$ will converge to $A$, that is, $\lim F^{n}(x)=A$.

Now, let us fix $t_{0} \in(0,1)$ and call $p=\gamma\left(t_{0}\right)$. For each $s \in\left[t_{0}, 1\right]$ we obtain the open ball $B\left(\gamma(s) ; \delta_{s}\right)$ where points will converge to the basin of attraction of $A$, as defined above. By compactness of $\gamma\left(\left[t_{0}, 1\right]\right)$, we can extract a finite subcover from the open cover $\left\{B\left(\gamma(s) ; \delta_{s}\right)\right\}$. Using the Lebesgue Covering Lemma, we are able to find $\eta_{p}>0$ and a tubular neighbourhood $T_{\eta_{p}}$ around $\gamma\left(\left[t_{0}, 1\right]\right)$ with the property that for each $x \in T_{\eta_{p}}$, we have $\lim F^{n}(x)=A$ as depicted in Figure 1 .

Next, we must extend the tubular neighbourhood beyond $\gamma\left(\left[t_{0}, 1\right]\right)$ to be a tubular neighbourhood of $\gamma$. As we approach the saddle point $S$ the neighbourhood $T_{\eta_{p}}$ could degenerate. Nevertheless, the local structure of $S$ will enable us to find the desired tubular neighbourhood around the heteroclinic connection. Indeed, using the Hartman-Grobman Theorem [27], the map $F$ is locally topologically conjugate to its derivative $J F$, that is, the Jacobian matrix of $F$. Hence, there is a neighbourhood $U$ of $S$ and a conjugate diffeomorphism $\varphi: U \rightarrow V$ such that $\varphi(U)=V \subseteq \mathbb{R}^{k}$ and $\varphi \circ F=J F \circ \varphi$ or equivalently $F=\varphi^{-1} \circ J F \circ \varphi$ as depicted in Diagram (3). Let us denote by $H^{+}$the half-space of $\mathbb{R}_{+}^{k}$ that contains $\varphi(\gamma)$. In what follows, all neighbourhoods are with respect to the subspace topology of $\mathrm{H}^{+}$.

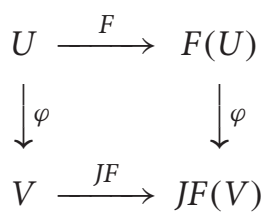

Let $t_{0} \in(0,1]$ with $p=\gamma\left(t_{0}\right) \in U$ and $\hat{p}=\varphi(p) \in V$, as depicted in Figure 2 , then choose $\delta>0$ such that $B(\hat{p} ; \delta) \subseteq \varphi\left(T_{\eta_{p}}\right)$. Next, for $s \in\left[0, t_{0}\right]$, denote $q=\gamma(s)$ and $\hat{q}=$ $\varphi(q)$. By the local structure of the linear system generated by $J F$, for any $\hat{z} \in B(\hat{q} ; \delta) \cap H^{+}$, we have that $J F^{m}(\hat{z}) \in B(\hat{p} ; \delta)$, for some $m>0$. This means that for any point $\hat{z}$ within a distance $\delta$ to $\hat{\gamma}_{t}=\varphi\left(\gamma\left(\left[0, t_{0}\right]\right)\right)$, the orbit of $\hat{z}$ under the linear system generated by $J F$ will eventually be in $B(\hat{p} ; \delta)$, which implies that the orbit of $z=\varphi^{-1}(\hat{z})$ will eventually be in $T_{\eta_{p}}$ and thus will converge to the attractor $A$.

Therefore, let $W=B\left(\hat{\gamma}_{t} ; \delta\right) \cap H^{+}$be the neighbourhood of $\hat{\gamma}_{t}$ as shown in Figure 2 . Then the set $G=T_{\eta_{p}} \cup \varphi^{-1}(W)$ is the desired neighbourhood of $\gamma$, where for $x \in G \cap \operatorname{int}\left(\mathbb{R}_{+}^{n}\right)$, $\lim F^{n}(x)=A$.

\section{Planar maps}

The concept of monotonicity introduced in Definition 2.2 may be easily stated for planar maps. Indeed, given a curve $\gamma:[0,1] \rightarrow \mathbb{R}^{2}$, we say that $\gamma$ is a monotone curve if for any $t \in(0,1)$, the normal vector $\eta(\gamma(t))$ is positive, that is, it belongs to the first quadrant. Since our primary concern is with the sign of the coordinates of the normal vector, we do not need to normalize $\eta(\gamma(t))$.

When we compute the normal vector, we are implicitly requiring that our curve $\gamma$ is differentiable. However, as we will soon observe, this is not needed as the main interest is on the geometry associated with monotonicity. In fact, we can see that positivity of the normal vector is simply a way to capture that the curve $\gamma$ is increasing with respect to the $x$-axis and decreasing with respect to the $y$-axis. Indeed, if $\gamma(t)=(\alpha(t), \beta(t))$, then 


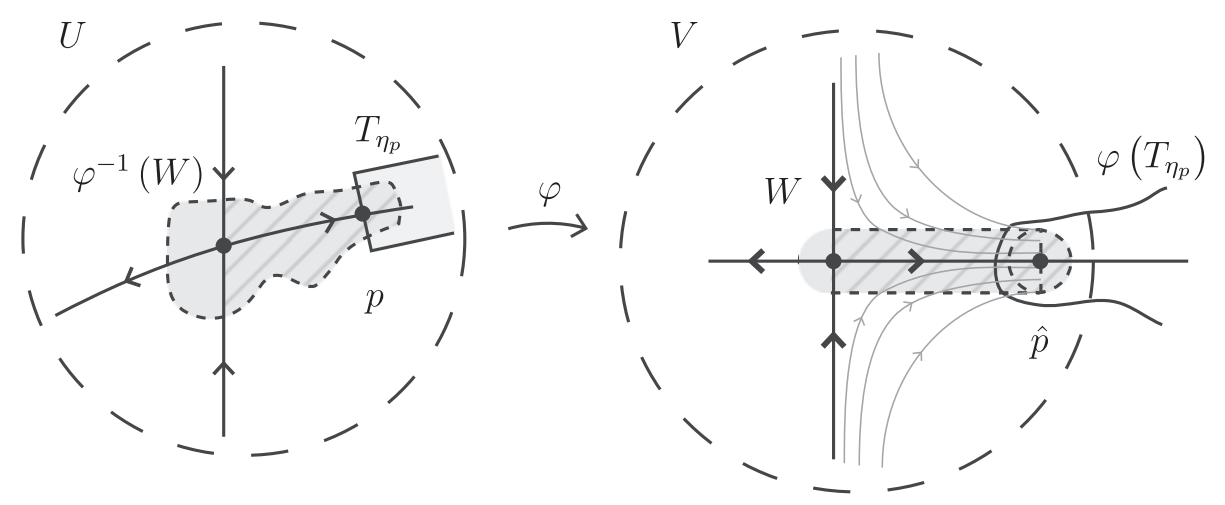

Figure 2. The local structure of a saddle point and its dynamics.

$\eta(\gamma(t))=\left(-\beta^{\prime}(t), \alpha^{\prime}(t)\right)$. Hence to say that $\gamma$ is a monotone curve is equivalent to say that $\alpha(t)$ is increasing and $\beta(t)$ is decreasing.

The discussion above essentially shows that for planar maps, our concepts of monotone maps and competitive maps are equivalent. Recall that a map $F: \mathbb{R}^{2} \rightarrow \mathbb{R}^{2}$ is called competitive (monotone in the sense of Smith [32]) if it preserves the southeast order, that is, the order induced by the fourth quadrant, to be denoted by $K$. Also, a domain $\Omega$ is said to be $K$-convex if it contains the line segment joining any two points that are ordered with respect to $K$.

Lemma 3.1: Let $\Omega$ be a $K$-convex region in $\mathbb{R}^{2}$ and $F: \Omega \rightarrow \mathbb{R}^{2}$ be a $C^{1}$ map. Then the map $F$ is monotone according to Definition 2.2 if and only if it is a competitive map.

Proof: First, let us assume that $F$ is competitive. Take a curve $\gamma(t)=\left(\alpha_{1}(t), \alpha_{2}(t)\right)$ with positive normal. This means that the map $\alpha_{1}$ is increasing and $\alpha_{2}$ is decreasing.

Next, let $F(\gamma(t))=\left(\beta_{1}(t), \beta_{2}(t)\right)$. In order to show that $F$ is monotone, we need to show that $\beta_{1}$ is increasing and $\beta_{2}$ is decreasing. Indeed, for $s<t$ we have that

$$
\alpha_{1}(s)<\alpha_{1}(t), \quad \alpha_{2}(s)>\alpha_{2}(t)
$$

Since $F$ is $K$-order preserving, we have

$$
\beta_{1}(s)<\beta_{1}(t), \quad \beta_{2}(s)>\beta_{2}(t) .
$$

Hence, this shows that $\beta_{1}$ is increasing and $\beta_{2}$ is decreasing, i.e. $F$ is monotone.

For the converse, let us assume that $F$ is monotone. Pick two points $\mathbf{x}=\left(x_{1}, x_{2}\right)$ and $\mathbf{y}=$ $\left(y_{1}, y_{2}\right)$ such that $\mathbf{x} \leq_{K} \mathbf{y}$. Consider the segment $\gamma(t)=\left((1-t) x_{1}+t y_{1},(1-t) x_{2}+t y_{2}\right)$ from $\mathbf{x}$ to $\mathbf{y}$. We can see that $\gamma^{\prime}(t)=\left(y_{1}-x_{1}, y_{2}-x_{2}\right)$ and hence $\eta(t)=\left(x_{2}-y_{2}, y_{1}-x_{1}\right)>0$.

Let $F(\gamma(t))=\left(\beta_{1}(t), \beta_{2}(t)\right)$. Since $F$ is monotone, it will satisfy that $\beta_{1}$ is increasing and $\beta_{2}$ is decreasing. Therefore, it is straightforward to see that $F(\mathbf{x}) \leq_{K} F(\mathbf{y})$ and thus $F$ is $K$-preserving.

It is an important problem to find an analytic condition to detect when a map is competitive or, equivalently, monotone. In fact, Smith [32, Proposition 2.1] has provided this condition. That is, $F$ is monotone if the Jacobian matrix $J F$ is a $K$-positive matrix. Here a matrix is said to be $K$-positive if its diagonal entries are positive and its off-diagonal 
entries are negative. We further observe that this is equivalent to say that the cofactors of the matrix are positive when the determinant is positive. We remark that Smith himself noted in [32] that these conditions are not the most general one can find, for example, one may consider nonnegative and nonpositive entries, and so forth. Indeed, one could consider preservation of nonnegative normals and modify the conditions on JF. Although this is an important consideration that can easily be addressed when actually checking for monotonicity, we believe that it will detract us from the geometric arguments and the appreciation of the novel geometric ideas. Thus, just as Smith [32] did, we will not consider it here.

It is a simple computation to show that if $J F$ is a $K$-positive matrix, then $F$ is monotone. Let $F\left(x_{1}, x_{2}\right)=\left(f_{1}\left(x_{1}, x_{2}\right), f_{2}\left(x_{1}, x_{2}\right)\right)$ be a $C^{1}$ map and assume that $J F$ is $K$-positive matrix, that is,

$$
J F(x, y)=\left(\begin{array}{ll}
\frac{\partial f_{1}}{\partial x_{1}} & \frac{\partial f_{1}}{\partial x_{2}} \\
\frac{\partial f_{2}}{\partial x_{1}} & \frac{\partial f_{2}}{\partial x_{2}}
\end{array}\right)
$$

has positive diagonal terms $\frac{\partial f_{1}}{\partial x_{1}}$ and $\frac{\partial f_{2}}{\partial x_{2}}$ and has negative off diagonal terms $\frac{\partial f_{1}}{\partial x_{2}}$ and $\frac{\partial f_{2}}{\partial x_{1}}$.

Let $\gamma$ be a monotone curve given by $\gamma(t)=\left(x_{1}(t), x_{2}(t)\right)$. The image of $\gamma$ under the map $F$ is given by

$$
F(\gamma(t))=\left(f_{1}\left(x_{1}(t), x_{2}(t)\right), f_{2}\left(x_{1}(t), x_{2}(t)\right)\right) .
$$

Now, we compute $\frac{\mathrm{d} F}{\mathrm{~d} t}$ and we have:

$$
\frac{\mathrm{d} F}{\mathrm{~d} t}=\left(\frac{\partial f_{1}}{\partial x_{1}} \frac{\mathrm{d} x_{1}}{\mathrm{~d} t}+\frac{\partial f_{1}}{\partial x_{2}} \frac{\mathrm{d} x_{2}}{\mathrm{~d} t}, \frac{\partial f_{2}}{\partial x_{1}} \frac{\mathrm{d} x_{1}}{\mathrm{~d} t}+\frac{\partial f_{2}}{\partial x_{2}} \frac{\mathrm{d} x_{2}}{\mathrm{~d} t}\right) .
$$

Since $\frac{\mathrm{d} x_{1}}{\mathrm{~d} t}>0$ and $\frac{\mathrm{d} x_{2}}{\mathrm{~d} t}<0$, we obtain,

$$
\frac{\partial f_{1}}{\partial x_{1}} \frac{\mathrm{d} x_{1}}{\mathrm{~d} t}+\frac{\partial f_{1}}{\partial x_{2}} \frac{\mathrm{d} x_{2}}{\mathrm{~d} t}>0 \text { and } \frac{\partial f_{2}}{\partial x_{1}} \frac{\mathrm{d} x_{1}}{\mathrm{~d} t}+\frac{\partial f_{2}}{\partial x_{2}} \frac{\mathrm{d} x_{2}}{\mathrm{~d} t}<0 .
$$

This shows that if $J F$ is a $K$-positive matrix, then the image of a monotone path is a monotone path. In fact, the computation above in (4) provides the analytic condition that one needs to verify condition (H2).

We are now ready to prove our main result in the particular case of planar maps. The proof in the planar case does not require the existence of the carrying simplex, in fact, we directly show its existence in this case. We include the proof here to highlight the geometric approach of our argument.

Theorem 3.2: $\quad$ Let $F: \Omega \rightarrow \Omega$ be a $C^{1}$ map. Assume that $F$ satisfies (H1)-(H3). If there is a unique positive fixed point $E^{*}$, then for every $\mathbf{p}>0, F^{n}(\mathbf{p}) \rightarrow E^{*}$ as $n \rightarrow \infty$, that is, $E^{*}$ is globally asymptotically stable in the interior of $\Omega$.

Proof: For simplicity, let $E_{\{i\}}=E_{i}$. Since $F$ satisfies (H2), it follows that Lemma 3.1 holds. Thus, $F$ is competitive and by a change of coordinate, say $(u, v) \mapsto(u,-v)$, the Jacobian matrix $J F$ becomes a positive matrix. By (H3), for $i=1,2$, we have that $\sigma\left(J F\left(E_{i}\right)\right)=$ $\left\{\lambda_{1}^{i}, \lambda_{2}^{i}\right\}$ with $\left|\lambda_{1}^{i}\right|<1<\left|\lambda_{2}^{i}\right|$.

Using the Perron-Frobenius Theorem, the associated eigenvector is positive. Changing it back to the original coordinates, the eigenvector will have coordinates with opposite 


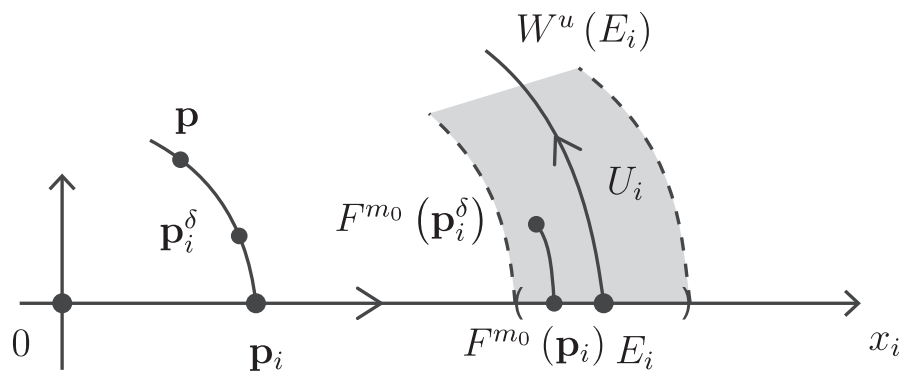

Figure 3. Points on the $x_{i}$-axis will converge to $E_{i}$. At a fixed iteration $m_{0}$, we can find an interior point $\mathbf{p}_{i}^{\delta}$ that will be contained in the neighbourhood $U_{i}$ after $m_{0}$ iterations.

signs. We then conclude that the locally unstable manifold of $E_{i}, W_{l o c}^{u}\left(E_{i}\right)$, is a normally monotone curve, and by $(\mathrm{H} 2)$, we have that $W^{u}\left(E_{i}\right)$ is a normally monotone curve as well. This means that $\left.F\right|_{W^{u}\left(E_{i}\right)}$ will be componentwise monotone and thus the orbits of all points in $W^{u}\left(E_{i}\right)$ must converge to a fixed point. By assumption, there is only one fixed point $E^{*}$, so orbits of points in $\left.F\right|_{W^{u}\left(E_{i}\right)}$ must converge to $E^{*}$.

Let $\mathbf{p} \in \Omega$, and without lost of generality, we can assume that $\mathbf{p} \in R$. Indeed, if that is not the case, then by (H1) we can find $\mathbf{q} \in R$ with $F(\mathbf{p})=F(\mathbf{q})$. Next, by hypotheses (H2), there is a curve $\gamma:[0,1] \rightarrow R$ that is monotone passing through $\mathbf{p}$ such that $\gamma(0)=\left(0, p_{2}\right)=\mathbf{p}_{2}$ and $\gamma(1)=\left(p_{1}, 0\right)=\mathbf{p}_{1}$.

We will show that there are points near the boundary of $\gamma, \mathbf{p}_{1}^{\delta}$ and $\mathbf{p}_{2}^{\delta}$ that will be attracted to $E^{*}$. More precisely, after $m$ iterations, their orbits will be contained in an $\varepsilon$-ball around $E^{*}$ denoted by $B\left(E^{*} ; \varepsilon\right)$. Since metrics are equivalent in a finite dimensional vector space, we can consider balls to be in the max norm, that is, balls are squares.

Since $E^{*}$ is a local attractor, let $B\left(E^{*} ; \varepsilon\right)$ be a subset of the immediate basin of attraction of $E^{*}$ contained in the basin of attraction of $E^{*}$. For each $i=1,2$, we have that $E_{i}$ is a saddle point and $E^{*}$ is a local attractor with a heteroclinic connection $W^{u}\left(E_{i}\right)$ between $E_{i}$ and $E^{*}$. Thus, by Lemma 2.7, there is a tubular neighbourhood $U_{i}$ of $W^{u}\left(E_{i}\right)$ such that for $\mathbf{x} \in U_{i} \cap \operatorname{int}\left(\mathbb{R}_{+}^{2}\right)$, we have $F^{n}(\mathbf{x}) \in B\left(E^{*} ; \varepsilon\right)$ for $n$ sufficiently large.

For each $i=1,2$, we have that $E_{i}$ is a globally asymptotically stable point of $F$ restricted to the axis $x_{i}$. Thus, there is $m_{i} \in \mathbb{N}$ such that for $n \geq m_{i}$, we have $F^{n}\left(\mathbf{p}_{i}\right) \in U_{i}$ as depicted in Figure 3. Let $m_{0}=\max \left\{m_{1}, m_{2}\right\}$ then for this fixed $m_{0} \in \mathbb{N} \mathbb{N}$ we have $F^{m_{0}}\left(\mathbf{p}_{i}\right) \in U_{i}$ for each $i=1,2$.

Since $F^{m_{0}}$ is a continuous map, there is a neighbourhood $V_{i}$ of $\mathbf{p}_{i}$, say $V_{i}=B\left(\mathbf{p}_{i} ; \delta_{i}\right)$ for $\delta_{i}>0$ such that if $\mathbf{q} \in V_{i}$, then $F^{m_{0}}(\mathbf{q}) \in U_{i}$. Let $\delta=\min \left\{\delta_{1}, \delta_{2}\right\}>0$ and choose $\mathbf{p}_{i}^{\delta} \in B\left(\mathbf{p}_{i} ; \delta\right) \cap \gamma$ and $\mathbf{p}_{i}^{\delta}$ is between $\mathbf{p}$ and $\mathbf{p}_{i}$ along $\gamma$. By the choice of $\delta$, we have that $F^{m_{0}}\left(\mathbf{p}_{i}^{\delta}\right) \in U_{i}$ as illustrated in Figure 3 .

Now, by the choice of $U_{i}$, we know that there is $n_{i} \in \mathbb{N}$ such that for $n \geq n_{i}$, we have $F^{n}\left(\mathbf{p}_{i}^{\delta}\right) \in B\left(E^{*} ; \varepsilon\right)$. Then choose $m=\max \left\{n_{1}, n_{2}\right\}$.

Because $\gamma$ is a monotone curve, the images of $\gamma$ under $F$ will be a graph of a function with respect to both axes. This means that for all $n \in \mathbb{N}$, the coordinates of $F^{n}(\mathbf{p})$ will lie between the coordinates of $F^{n}\left(\mathbf{p}_{1}^{\delta}\right)$ and $F^{n}\left(\mathbf{p}_{2}^{\delta}\right)$ as depicted in Figure 4. More precisely as $\gamma$ is a monotone curve increasing in the $x_{1}$-direction and decreasing in the $x_{2}$-direction:

$$
\left.\pi_{1}\left(F^{n}\left(\mathbf{p}_{2}^{\delta}\right)\right) \leq \pi_{1}\left(F^{n}(\mathbf{p})\right)\right) \leq \pi_{1}\left(F^{n}\left(\mathbf{p}_{1}^{\delta}\right)\right)
$$




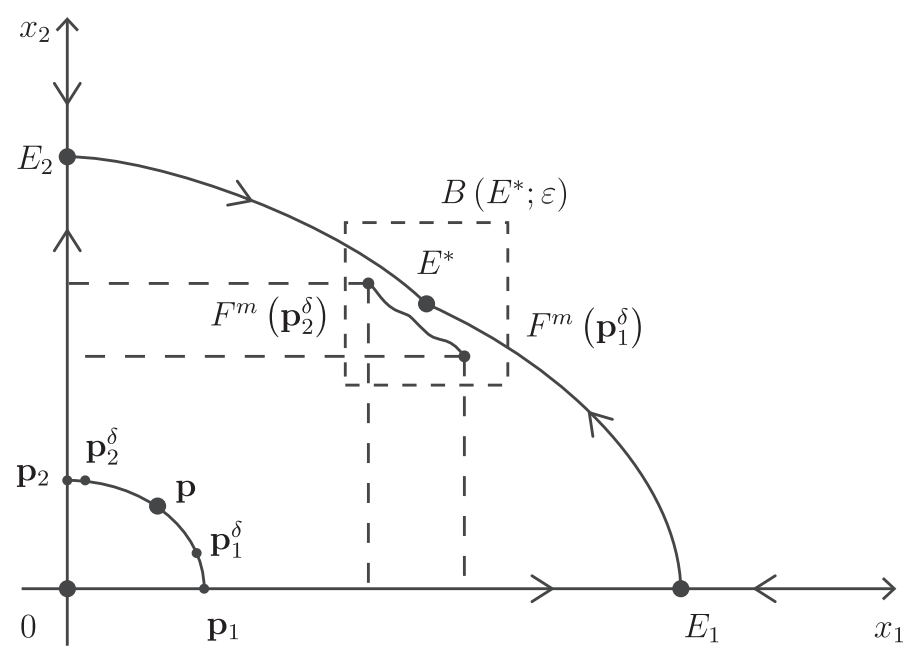

Figure 4. Dynamics of the image of a monotone path. The endpoints will converge to $E_{i}$ and points near the axes will converge to $E^{*}$. Monotonicity of $F$ ensures that the image of the path will also converge to $E^{*}$.

and

$$
\left.\pi_{2}\left(F^{n}\left(\mathbf{p}_{2}^{\delta}\right)\right) \geq \pi_{2}\left(F^{n}(\mathbf{p})\right)\right) \geq \pi_{2}\left(F^{n}\left(\mathbf{p}_{1}^{\delta}\right)\right) .
$$

For $m$ as chosen above, we have that $F^{m}\left(\mathbf{p}_{i}^{\delta}\right) \in B\left(E^{*} ; \varepsilon\right)$, so it must be that $F^{m}(\mathbf{p})$ must be contained in a square of size $\varepsilon$ centered at $E^{*}$ as depicted in Figure 4 .

Since the argument is valid for any $\varepsilon>0$, we have that $F^{n}(\mathbf{p}) \rightarrow E^{*}$ as $n \rightarrow \infty$ as we wanted to show.

Before end this section, we remark that the argument used in the proof that the restriction of $F$ to the unstable manifold is componentwise monotone can be stated in general for planar retrotone maps as follows.

Proposition 3.3: Suppose $F: \mathbb{R}_{+}^{2} \rightarrow \mathbb{R}_{+}^{2}$ is a retrotone map and $E$ is a saddle fixed point. If $W_{\text {loc }}^{u}(E)$ is not contained in the positive or negative cone centered at $E$, then the restriction of $F$ to $W^{u}(E)$ is componentwise monotone.

Proof: Let $\sigma(J F(E))=\left\{\lambda_{1}, \lambda_{2}\right\}$ with $\left|\lambda_{1}\right|<1<\left|\lambda_{2}\right|$ and $v_{2}$ be the eigenvector associated to $\lambda_{2}$. Thus $W_{\text {loc }}^{u}(E)$ is locally in the same direction as $v_{2}$. Hence to say that $W_{l o c}^{u}(E)$ is contained in the positive or negative cone is equivalent to say that the coordinates of $v_{2}$ have the same sign.

Next, we see that $W^{u}(E)$ can be viewed as a smooth curve as it is the image of $W_{l o c}^{u}(E)$ under $F$ which is a smooth curve. We will prove our result by establishing the contrapositive. Indeed, suppose that the restriction of $F W^{u}(E)$ is not a componentwise monotone. Then, there is $\mathbf{x} \in W^{u}(E)$ where the curve $W^{u}(E)$ changes direction. This means that the curve $W^{u}(E)$ will stay in the same half-plane if we consider $\mathbf{x}$ as the origin. Thus, we can find $\mathbf{y} \in W^{u}(E)$ in one of the quadrants such that $\mathbf{x}$ and $\mathbf{y}$ are ordered with respect to the usual order, that is, without loss of generality, say $\mathbf{y}>\mathbf{x}$.

Since $F$ is retrotone, we have that $F^{-1}(\mathbf{y})>F^{-1}(\mathbf{x})$ and because $W^{u}(E)$ is invariant $F^{-1}(\mathbf{y}), F^{-1}(\mathbf{x}) \in W^{u}(E)$. For $n \in \mathbb{N}$ sufficiently large $F^{-n}(\mathbf{y}), F^{-n}(\mathbf{x}) \in W_{l o c}^{u}(E)$ and 
$F^{-n}(\mathbf{y})>F^{-n}(\mathbf{x})$. Hence $W_{\text {loc }}^{u}(E)$ must be contained in the positive or negative cone centered at $E$.

\section{Normally monotone maps in $\mathbb{R}^{k}$}

In this section, we discuss how our geometric definition of monotonicity in terms of preservation of the direction of the normal vector is the appropriate generalization of the results from planar maps to higher dimensional maps and provides a generalization of competitive maps in higher dimensions.

Let us recall some basic terminology from Linear Algebra to facilitate the description of our results. Given a $k \times k$ matrix $M$, for $1 \leq i, j \leq k$, let $M_{i}$ be the $(k-1) \times k$ matrix obtained from $M$ by deleting its $i$ th row and $M^{j}$ be the $k \times(k-1)$ matrix obtained from $M$ by deleting its $j$ th column. Since in the sequel we will not consider power of matrices, this notation should not cause any ambiguity. Thus we denote $\operatorname{det} M_{i}^{j}$ to be the $(i, j)$-minor and $C_{i j}(M)=(-1)^{i+j} \operatorname{det} M_{i}^{j}$ to be the $(i, j)$-cofactor.

In the previous section, we showed that if the Jacobian matrix of a planar map is $K$ positive, then the map is normally monotone. In higher dimensions, the analogous result is true. However, one should not be looking at the signs of individual entries of the Jacobian matrix. Instead, one should look at the sign of the cofactors of the Jacobian matrix. Observe that in the plane, when the Jacobian matrix is $2 \times 2$, the sign of the cofactors is given by the sign of the corresponding entries. Our result is as follows.

Lemma 4.1: Let $\Omega \subset \mathbb{R}^{k}$ and $F=\left(f_{1}, \ldots, f_{k}\right): \Omega \rightarrow \mathbb{R}^{k}$ be a map of class $C^{1}$. Let $\mathbf{p}$ be a regular value. The cofactors of the Jacobian matrix $J F(\mathbf{p})$ are positive if and only if $F$ is normally monotone at $\mathbf{p}$.

We remark that the requirement that $\mathbf{p}$ is a regular value, i.e. $\operatorname{det} J F(\mathbf{p}) \neq 0$, is simply to ensure that the tangent space and normal vector are well-defined. Note that for a local orientation preserving diffeomorphism $F$ of class $C^{1}$, if $J F^{-1}(\mathbf{p})$ is a positive matrix, then $F$ is normally monotone. This is precisely the conditions from Smith's original work on competitive maps in [31] where the condition of positivity and irreducibility of a matrix was used to verify the hypotheses of the Perron-Frobenius Theorem. From our geometric approach, one of our contributions is to use the positivity of $J F^{-1}$ to show that $F$ is componentwise monotone when restricted to the one-dimensional unstable manifold of the fixed points in the coordinates hyperplanes. This will be formally stated in Lemma 4.3.

For now, we proceed with the outline of the proof of Lemma 4.1 which will be a simple computation. Namely, starting with a hypersurface with positive normal vector, we compute the normal vector to the image. Then, we show that the coordinate of the normal vector is given by summands that involve the cofactors of the Jacobian matrix. The terms in the summand will appear from the classical Cauchy-Binet formula [22] which states that if $A, B$ are two matrices of sizes $m \times k, k \times m$, respectively, with $m \leq k$, then

$$
\operatorname{det}(A B)=\sum_{\sigma} \operatorname{det}\left(A^{\sigma}\right) \operatorname{det}\left(B_{\sigma}\right) .
$$

The sum is taken over all $\sigma=\left(\sigma_{1}<\sigma_{2}<\cdots<\sigma_{m}\right)$, with $\sigma_{i} \in\{1, \ldots, k\}$, where $A^{\sigma}$ (and $B_{\sigma}$ ) is the submatrix of $A$ (and the submatrix of $B$ ) obtained by deleting all columns (all rows) except those with indices in $\sigma$. 
Proof of Lemma 4.1: Consider a hypersurface $\Gamma \subseteq \Omega$ and $\mathbf{p} \in \Gamma$ such that $\eta_{\Gamma}(\mathbf{p})>0$, that is, the normal vector at $\mathbf{p}$ is a positive vector. Let the local coordinates of $\Gamma$ be given by $\Gamma: \mathbb{R}^{k-1} \rightarrow \mathbb{R}^{k}$ where

$$
\Gamma\left(u_{1}, \ldots, u_{k-1}\right)=\left(x_{1}\left(u_{1}, \ldots, u_{k-1}\right), \ldots, x_{k}\left(u_{1}, \ldots, u_{k-1}\right)\right) .
$$

Thus the tangent space of $\Gamma$ at $\mathbf{p}$ is spanned by the column vectors of the matrix

$$
D \Gamma=\left(\begin{array}{ccc}
\frac{\partial x_{1}}{\partial u_{1}} & \cdots & \frac{\partial x_{1}}{\partial u_{k-1}} \\
\vdots & \ddots & \vdots \\
\frac{\partial x_{k}}{\partial u_{1}} & \cdots & \frac{\partial x_{k}}{\partial u_{k-1}}
\end{array}\right)
$$

Therefore, the normal vector $\eta=\eta_{\Gamma}(\mathbf{p})$ may be computed as

$$
\eta=\sum_{i=1}^{k}\left((-1)^{1+i} \operatorname{det}\left(\left(D \Gamma^{T}\right)^{i}\right)\right) \mathbf{e}_{i}=\sum_{i=1}^{k}\left((-1)^{1+i} \operatorname{det}\left(D \Gamma_{i}\right)\right) \mathbf{e}_{i}
$$

Considering the coordinates of $\eta=\left(\eta_{1}, \ldots, \eta_{k}\right)$, from the assumption that $\eta>0$, we have for $i=1,2, \ldots, k$ that

$$
\eta_{i}=(-1)^{1+i} \operatorname{det}\left(D \Gamma_{i}\right)>0
$$

Now, we consider the image of $\mathbf{p}$ and the normal vector at $F(\mathbf{p})$ to $F(\Gamma)$. First, the tangent space is spanned by vectors $F_{u_{1}}, \ldots, F_{u_{k-1}}$ where

$$
F_{u_{j}}=\left(\frac{\partial f_{1}}{\partial u_{j}}, \ldots, \frac{\partial f_{k}}{\partial u_{j}}\right)
$$

From the chain rule, we have that

$$
\frac{\partial f_{i}}{\partial u_{j}}=\sum_{r=1}^{k} \frac{\partial f_{i}}{\partial x_{r}} \frac{\partial x_{r}}{\partial u_{j}}
$$

which is the $(i, j)$-entry of $J F \cdot D \Gamma$. Next, we compute the normal vector at the $F(\mathbf{p})$, denoted by $\eta_{F}=\eta_{F(\Gamma)}(F(\mathbf{p}))$, and obtain

$$
\begin{aligned}
\eta_{F} & =\sum_{i=1}^{k}\left((-1)^{1+i} \operatorname{det}\left(\left[(J F \cdot D \Gamma)^{T}\right]^{i}\right)\right) \mathbf{e}_{i} \\
& =\sum_{i=1}^{k}\left((-1)^{1+i} \operatorname{det}\left((J F \cdot D \Gamma)_{i}\right)\right) \mathbf{e}_{i} .
\end{aligned}
$$


Observe that $(J F \cdot D \Gamma)_{i}=J F_{i} \cdot D \Gamma$ where $J F_{i}$ is a $(k-1) \times k$ matrix and $D \Gamma$ is an $k \times(k-1)$ matrix. Now, using the Cauchy-Binet formula (5), we obtain:

$$
\operatorname{det}\left(J F_{i} \cdot D \Gamma\right)=\sum_{j=1}^{k} \operatorname{det}\left(J F_{i}^{j}\right) \operatorname{det}\left(D \Gamma_{j}\right)
$$

Hence the $i$ th coordinate of $\eta_{F}$ is given by

$$
\begin{aligned}
\left(\eta_{F}\right)_{i} & =\sum_{j=1}^{k}(-1)^{1+i} \operatorname{det} J F_{i}^{j} \cdot \operatorname{det} D \Gamma_{j} \\
& =\sum_{j=1}^{k}(-1)^{i+j} \operatorname{det} J F_{i}^{j} \cdot(-1)^{1+j} \operatorname{det} D \Gamma_{j} \\
& =\sum_{j=1}^{k} C_{i j}(J F) \cdot \eta_{j}>0 .
\end{aligned}
$$

From (8), we directly verify that if all the cofactors of the Jacobian matrix $J F(\mathbf{p})$ are positive, then $F$ is monotone. Indeed, if $\eta>0$, then each $i$ th coordinate of $\eta_{F}$ is also positive.

In the other direction, assume $F$ is monotone. Assume towards a contradiction that there is one cofactors of the Jacobian matrix $J F(\mathbf{p})$ that is negative. Observe that given $a_{1}, a_{2}, \ldots, a_{k} \in \mathbb{R}$, if at least one $a_{i}$ is negative, then there exists $x_{1}, \ldots, x_{k}>0$ with $\sum x_{i}^{2}=1$ such that $\sum a_{i} x_{i}<0$. From this observation, we can choose a hypersurface $\Gamma$ nearly parallel to a coordinate hyperplane so that (8) is negative, a contradiction.

This establishes that the geometric hypotheses that $F$ is normally monotone may be characterized by the analytic condition that the cofactors of the Jacobian matrix $J F(\mathbf{p})$ are positive.

We highlight that similarly to the planar case, condition $(\mathrm{H} 2)$ can be analytically verified using Lemma 4.1. This allow us to show via a simple computation that $F$ is retrotone. Indeed, this is also found in [31, Proposition 2.3] and [17, Proposition 4.1] and we include it here for completeness.

Lemma 4.2: Let $F: \Omega \rightarrow \Omega$ be a $C^{1}$ that satisfies $(\mathrm{H} 1)-(\mathrm{H} 2)$. Then $F$ is retrotone.

Proof: Let us consider $\mathbf{x}, \mathbf{y} \in R$. Take a path in $F(R)$ joining $F(\mathbf{x})$ and $F(\mathbf{y})$ given by $\beta(t)=t F(\mathbf{y})+(1-t) F(\mathbf{x})$ for $t \in[0,1]$. Further, assume that

$$
\alpha(t)=F^{-1}(t F(\mathbf{y})+(1-t) F(\mathbf{x}))
$$

is well-defined. Since $F(\alpha(t))=\beta(t)$, we may differentiate both sides to obtain $J F(\alpha(t)) \alpha^{\prime}(t)=F(\mathbf{y})-F(\mathbf{x})$. That is,

$$
\alpha^{\prime}(t)=[J F(\alpha(t))]^{-1}(F(\mathbf{y})-F(\mathbf{x}))
$$


Now, we can integrate both sides of (9) to obtain

$$
\int_{0}^{1} \alpha^{\prime}(t) \mathrm{d} t=\alpha(1)-\alpha(0)=\mathbf{y}-\mathbf{x} .
$$

Thus by (9),

$$
\mathbf{y}-\mathbf{x}=\left(\int_{0}^{1}[J F(\alpha(t))]^{-1} \mathrm{~d} t\right)(F(\mathbf{y})-F(\mathbf{x})) .
$$

Since the map $F$ is monotone, we have by Lemma 4.1 that $J F^{-1}$ is a positive matrix. Therefore if $F(\mathbf{y})>F(\mathbf{x})$, then (10) implies that $\mathbf{y}>\mathbf{x}$. Hence $F$ is a retrotone map.

Next, we state the result mentioned above that establishes componentwise monotonicity of $F$ when restricted to the one dimensional unstable manifold.

Lemma 4.3: Assume that the map $F: \Omega \rightarrow \Omega$ satisfies $(\mathrm{H} 1)-(\mathrm{H} 4)$. Then for each $I \subset$ $\{1, \ldots, k\}$ with $|I|=k-1$, the restriction map $\left.F\right|_{W^{u}\left(E_{I}\right)}$ is componentwise monotone.

Without loss of generality, let us consider $I=\{1, \ldots, k-1\}$ and denote $E_{I}=E_{k}$. From hypothesis (H4), $F$ admits a carrying simplex $\Sigma$ and $W^{u}\left(E_{k}\right) \subseteq \Sigma$.

Since $F$ is monotone, and thus preserves positive normal vectors, the carrying simplex $\Sigma$ has positive normals at each point. Indeed, $\Sigma$ may be approximated by the image of a boundary of a disk around the origin inside the positive cone. Hence, $\Sigma$ can be viewed as a graph of a function over each hyperplane. More importantly, for each $i \in I$, the projection map $\pi_{i k}: \Sigma \rightarrow H_{i k}$ admits a right inverse $\pi_{i k}^{-1}$.

Now, let us consider the dynamics of $W^{u}\left(E_{k}\right) \subseteq H_{i k}$ using the planar map $\tilde{F}: H_{i k} \rightarrow$ $H_{i k}$ as follows

$$
\begin{array}{ccc}
\Sigma \stackrel{F}{\longrightarrow} & \Sigma \\
\uparrow_{i k}^{-1} & & \downarrow \pi_{i k} \\
H_{i k} \stackrel{\tilde{F}}{\longrightarrow} & H_{i k}
\end{array}
$$

Note that because the carrying simplex is invariant, we can reduce the analysis of the componentwise monotonicity of $\left.F\right|_{W^{u}\left(E_{k}\right)}$ to the dynamics of a planar map $\tilde{F}$. First, observe that $\tilde{F}$ is a monotone map. This follows directly from the structure of the Jacobian matrix for the projection maps which are the $i$ and $k$ columns of the identity matrix. Thus, by the chain rule, it is clear to see that $J \tilde{F}^{-1}>0$ as it is the product of positive matrices.

Now, we can use Proposition 3.3 to show that $\left.F\right|_{W^{u}\left(E_{k}\right)}$ is componentwise monotone in the components $i$ and $k$. First, we see that $\pi_{i k}\left(W^{u}\left(E_{k}\right)\right)$ is an invariant subset under $\tilde{F}$ as $W^{u}\left(E_{k}\right)$ is invariant under $F$. Next, the normal vector to $\pi_{i k}\left(W^{u}\left(E_{k}\right)\right)$ at $\pi_{i k}\left(E_{k}\right)$ is positive since it is the projection of the normal vector to $W^{u}\left(E_{k}\right)$ which is positive. Thus the tangent vector to $\pi_{i k}\left(W^{u}\left(E_{k}\right)\right)$ at $\pi_{i k}\left(E_{k}\right)$ has opposite coordinates. The argument in Proposition 3.3 can then be used to conclude that $\left.F\right|_{W^{u}\left(E_{k}\right)}$ is componentwise monotone in the components $i$ and $k$.

By repeating this argument for each $i \in I$, we establish that $\left.F\right|_{W^{u}\left(E_{k}\right)}$ is componentwise monotone.

The significance of Lemma 4.3 is that when considering the dynamics of a monotone map restricted to the one dimensional unstable manifold, the map is componentwise monotone and the orbits in $W^{u}\left(E_{I}\right)$ must converge to a fixed point. In fact, as we consider 
the orbits in $W^{u}\left(E_{I}\right)$, we observe that they must be contained in the stable manifold of another fixed point, that is, we will have a heteroclinic connection. Moreover, if we know the nature of the stability of the two fixed points, say between a saddle and an attractor, then as shown in Lemma 2.7 points near the heteroclinic connection will be in the immediate basin of attraction of the stable fixed point. We are now ready to prove our main result, which we restate it here.

Theorem 2.4: Assume that $F: \Omega \rightarrow \Omega$ satisfies (H1)-(H4). If $F$ has a unique positive coexistence fixed point $E^{*}$, then $E^{*}$ is globally asymptotically stable in the interior of $\Omega$.

Proof: Consider $\mathbf{p} \in \Omega$ and without loss of generality we can assume that $\mathbf{p} \in R$. Indeed, if that is not the case, then by (H1) we can find $\mathbf{q} \in R$ with $F(\mathbf{p})=F(\mathbf{q})$. Next, choose $\Gamma$ to be a normally monotone hypersurface containing $\mathbf{p}$. Indeed, $\Gamma$ can be chosen to be the boundary of a ball at the origin or a translation of the boundary of $R$. We will show that the orbit of $\mathbf{p}$ will converge to the interior fixed point $E^{*}$ by showing that for any $\varepsilon>0$ so that $B\left(E^{*} ; \varepsilon\right)$ is contained in the local basin of attraction of $E^{*}$, the orbit of $\mathbf{p}$ will eventually be inside $B\left(E^{*} ; \varepsilon\right)$.

For each $r=1,2, \ldots k$, let $I_{r}=\{1,2, \ldots, k\}-\{r\}$ and consider the fixed point $E_{I_{r}}$. By hypotheses (H3), $E_{I_{r}}$ is a saddle point which is globally stable for the restriction map $\left.F\right|_{H_{I_{r}}}$. This means that we have a one dimensional global unstable manifold $W^{u}\left(E_{I_{r}}\right)$. From Lemma 4.3 , we conclude that any orbit in $W^{u}\left(E_{I_{r}}\right)$ will converge to an interior fixed point, in fact the unique fixed point $E^{*}$.

The argument above shows that $W^{u}\left(E_{I_{r}}\right)$ is a heteroclinic connection between $E_{I_{r}}$ and $E^{*}$. Thus, by Lemma 2.7 we can find a tubular neighbourhood $U_{r}$ of $W^{u}\left(E_{I_{r}}\right)$ so that each point $\mathbf{x} \in U_{r} \cap \operatorname{int}\left(\mathbb{R}_{+}^{k}\right)$, we have $F^{n}(\mathbf{x}) \in B\left(E^{*} ; \varepsilon\right)$ for $n$ sufficiently large.

Before we proceed, we will briefly outline the geometric idea of the proof. We will show that there it will be possible to find a small retraction of the boundary of $\Gamma$ so that the orbits of the boundary points will be contained in the basin of attraction of $E^{*}$. The main challenge, and hence the introduction of some heavier notation, is to find one fixed $m \in \mathbb{N}$ so that after $m$ iterations the boundary will be contained in $B\left(E^{*} ; \varepsilon\right)$. We will find finitely many anchor points that will be mapped to $B\left(E^{*} ; \varepsilon\right)$. Then, similarly to the planar proof of Theorem 3.2, the boundary monotone sets will be mapped to $B\left(E^{*} ; \varepsilon\right)$ and will remain monotone (or graphs of functions over hyperplanes). This implies that the interior of the monotone sets will also be contained in $B\left(E^{*} ; \varepsilon\right)$.

Let us now begin introducing some notation and providing the details of this argument. First, let us denote $\gamma=\partial \Gamma$ and $\overrightarrow{\mathbf{e}}_{r}$ to be the ray from the origin to the fixed point $E_{I_{r}}$. We define $\mathbf{p}_{r}=\overrightarrow{\mathbf{e}}_{r} \cap \gamma \in H_{I_{r}}$ which is an interior point of $H_{I_{r}}$. By (H3), the orbit of $\mathbf{p}_{r}$ converges to $E_{I_{r}}$ and we can find $m_{r} \in \mathbb{N}$ such that for $n \geq m_{r}, F^{n}\left(\mathbf{p}_{r}\right) \in U_{r}$. Now, let $m_{0}=\max \left\{m_{1}, m_{2}, \ldots, m_{k}\right\}$ and then for a fixed $m_{0}$, whenever $n \geq m_{0}, F^{n}\left(\mathbf{p}_{r}\right) \in U_{r}$ as illustrated in Figure 5.

Using that $F^{m_{0}}$ is continuous, we can find $\delta_{r}>0$ so that for each $\mathbf{x} \in B\left(\mathbf{p}_{r} ; \delta_{r}\right)$ we have that $F^{m_{0}}(\mathbf{x}) \in U_{r}$. Then let $\delta=\min \left\{\delta_{1}, \delta_{2}, \ldots, \delta_{k}\right\}>0$ and define $\Gamma^{\delta}=\{\mathbf{x} \in$ $\Gamma \mid \operatorname{dist}(\mathbf{x}, \gamma) \geq \delta / 2\}$, that is, $\Gamma^{\delta}$ is a small retraction of $\Gamma$ away from the boundary and $\gamma^{\delta}=\partial \Gamma^{\delta}$. Now we can select a anchor point $\mathbf{p}_{r}^{\delta} \in \Gamma^{\delta} \cap B\left(\mathbf{p}_{r} ; \delta\right)$, that is, a point in the interior of $\mathbb{R}_{+}^{k}$ in $\Gamma$ within $\delta$ from $\mathbf{p}_{r}$ such that $F^{m_{0}}\left(\mathbf{p}_{r}^{\delta}\right) \in U_{r}$. 


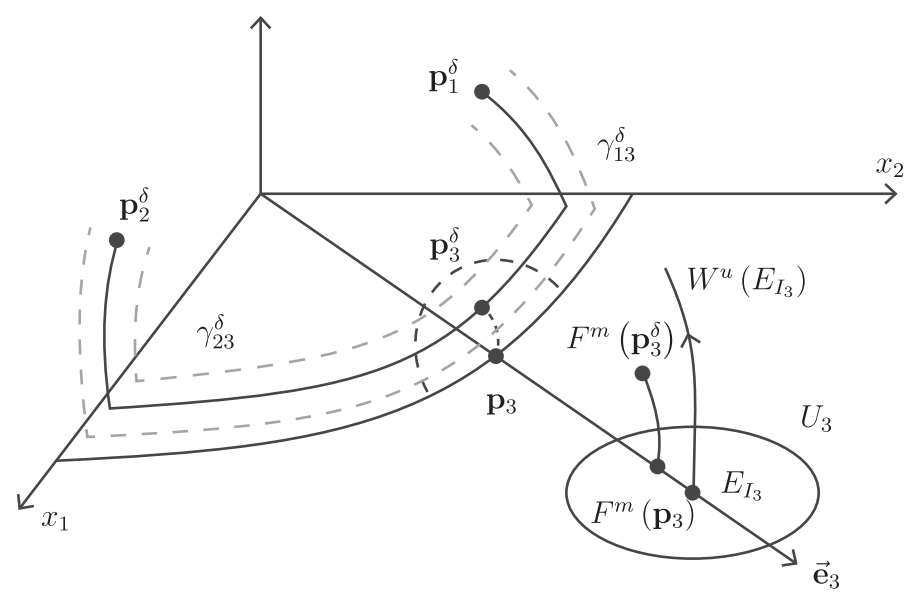

Figure 5. At each hyperplane, we can choose anchor points $\mathbf{p}_{r}^{\delta}$ that after $m_{0}$ iterations will be contained in $U_{r}$ and thus eventually attracted to $E^{*}$. It is also indicated how the one dimensional boundary above is decomposed into the 1 -cells $\gamma_{13}^{\delta}$ and $\gamma_{23}^{\delta}$.

Therefore, the orbit of $\mathbf{p}_{r}^{\delta}$ will eventually be contained in $B\left(E^{*} ; \varepsilon\right)$ and because there are finitely many of these anchor points $\mathbf{p}_{r}^{\delta}$, there is a fixed $m \in \mathbb{N}$ such that $F^{m}\left(\mathbf{p}_{r}^{\delta}\right) \in B\left(E^{*} ; \varepsilon\right)$ for all $r=1,2, \ldots k$. Indeed, for each $k$, we can find the first iteration where $\mathbf{p}_{r}^{\delta}$ will be contained in $B\left(E^{*} ; \varepsilon\right)$ and select $m$ to be the maximum of these iterations.

Now, we will reassemble $\Gamma^{\delta}$ by considering its cell complex (CW) topological decomposition from zero dimensional cells up to $k$-dimensions, see [15] for details. In fact, by [7], we know that $\Gamma^{\delta}$ is topologically a $k$-cell and so we can use the notation and combinatorial approach in [4] to complete the reassembly. First, we begin with the 0 -cells of $\Gamma^{\delta}$ to be the points $\mathbf{p}_{r}^{\delta}$. For notational purposes, we let $\gamma_{\{r\}}^{\delta}=\mathbf{p}_{r}^{\delta}$. Next, for $1 \leq \ell \leq k-1$, we consider all the subsets $J \subseteq\{1,2, \ldots k\}$ with cardinality $\ell$, that is, $|J|=\ell$. For each of these subsets, we will have a $\ell$-cell $\gamma_{J}^{\delta}$ such that the CW decomposition of the boundary will be as follows:

$$
\partial \gamma_{J}^{\delta}=\sum_{S \subset J,|S|=\ell-1} \gamma_{S}^{\delta} .
$$

In Figure 5 we illustrate this decomposition for the three dimensional case when $\Gamma^{\delta}$ is a two dimensional complex with a one dimensional boundary $\gamma^{\delta}$. In general, we start with the 0 -cells of $\Gamma^{\delta}$, that is, the $k$ points $\mathbf{p}_{r}^{\delta}$, will belong to $B\left(E^{*} ; \varepsilon\right)$ after $m$ iterations. Next, assume that for $0 \leq \ell<k-1$, all the $\ell$-cells of $\Gamma^{\delta}$ will belong to $B\left(E^{*} ; \varepsilon\right)$ after $m$ iterations. Then for $\ell+1$ and by $(12)$ the boundary of a $(\ell+1)$-cell $\gamma_{J}^{\delta}$ will belong to $B\left(E^{*} ; \varepsilon\right)$ after $m$ iterations. By monotonicity of $F$, the graph condition will be preserved and the $(\ell+1)$-cell must also be contained in $B\left(E^{*} ; \varepsilon\right)$ as depicted in Figure 6 for the three dimensional case. We do this up to $\ell=k-2$ to show that $\Gamma^{\delta}$, the $k-1$-cell is also eventually contained in $B\left(E^{*} ; \varepsilon\right)$.

Indeed, consider the projection of $\gamma_{J}^{\delta}$ onto each hyperplane of $\mathbb{R}_{+}^{k}$, say for instance $H_{I_{r}}$, and the continuous function $d_{r}$ defined to be the distance for $\pi_{r}(\mathbf{q})$ to $\pi_{r}\left(E^{*}\right)$ in $H_{I_{r}}$ for $\mathbf{q} \in \gamma_{J}^{\delta}$. By compactness, this function has a maximum which must be contained in $\pi_{r}\left(B\left(E^{*} ; \varepsilon\right)\right)$. Indeed, suppose this were not the case, then the maximum would have to 


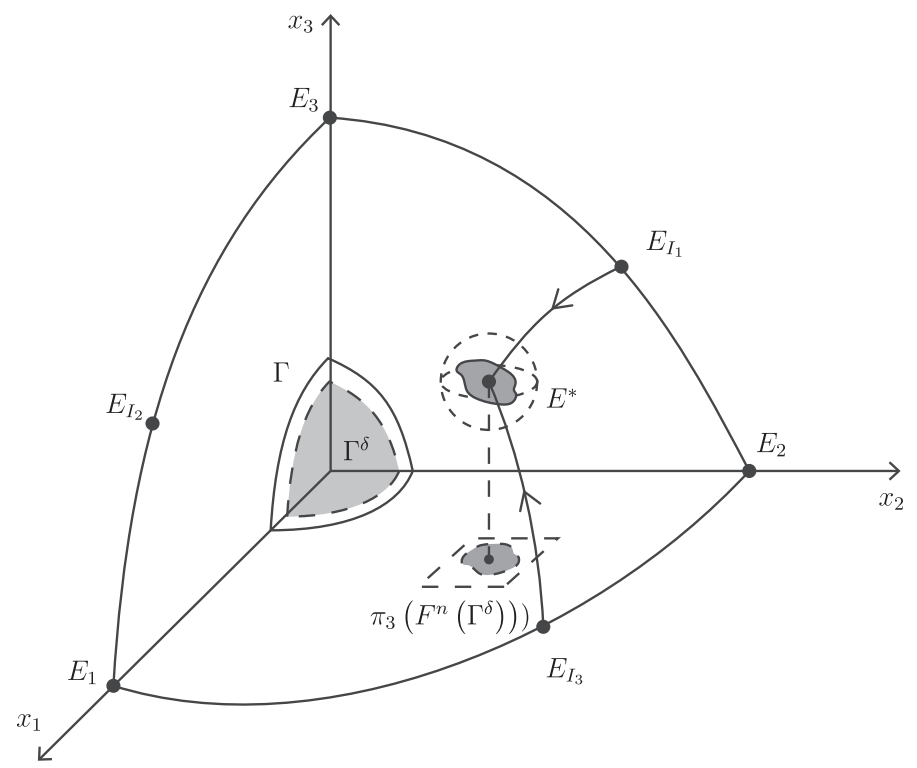

Figure 6. Dynamic of the image of the normally monotone hypersurface. The points near the boundary will converge to the interior fixed point $E^{*}$. The monotonicity of the map ensures the images will be a graph over each hyperplane and hence the images will belong to an $\varepsilon$-cube centered at $E^{*}$.

be at an interior point of $\gamma_{J}^{\delta}$. Therefore, a component, namely the $r$-component, of the normal vector of points in $\gamma_{J}^{\delta}$ would have to change sign and this would contradict the monotonicity of $F$.

Finally, iterating the argument until the top dimensional cell of $\Gamma^{\delta}$, we conclude that after $m$ iterations $\Gamma^{\delta}$ will be contained in the immediate basin of attraction of $E^{*}$, namely $B\left(E^{*} ; \varepsilon\right)$. In particular, the orbit of $\mathbf{p}$ must belong to $B\left(E^{*} ; \varepsilon\right)$. Since this argument is true for any $\varepsilon>0$, we have that $E^{*}$ is globally asymptotically stable as we wanted to prove.

\section{Applications}

In this section, we illustrate how to verify and apply our results to two models. We will consider the Leslie-Gower competition model used by Park and Leslie [25] and the Ricker Competition model [29]. We will first discuss the planar case and then address the three dimensional case. Although the planar results are known in the area, our main goal is to illustrate how to check the hypotheses $(\mathrm{H} 1)-(\mathrm{H} 4)$. We remark that the work in [17] has already shown that the Leslie-Gower and Ricker competition models admits a carrying simplex. Hence, since (H4) is verified for these two models, our main concerned here is to check (H1)-(H3). In addition, the global stability of the monotone Ricker planar map is often refereed in the literature to the work of Smith [32]. Unfortunately, the proof relies on a result, [32, Theorem 5.3], that does not have a published proof hence we included it here for completeness. 


\subsection{Leslie-Gower model}

The planar Leslie-Gower competition model is given by the following autonomous difference equation:

$$
\left\{\begin{array}{l}
x_{1}(n+1)=\frac{b_{1} x_{1}(n)}{1+c_{11} x_{1}(n)+c_{12} x_{2}(n)} \\
x_{2}(n+1)=\frac{b_{2} x_{2}(n)}{1+c_{21} x_{1}(n)+c_{22} x_{2}(n)}
\end{array}, n \in \mathbb{Z}^{+} .\right.
$$

The model has been rescaled to reduce the number of parameters, here $x_{1}(n)$ and $x_{2}(n)$ represents the population size of two species $x_{1}$ and $x_{2}$, respectively, at time unit $n$. The parameters $c_{i j}, i \neq j$ are the interspecific competition parameters, $c_{i i}$ are the intraspecific competition parameters and $b_{i}, i=1,2$ are the intrinsic growth rates. All these parameters are assumed to be positive constants. The asymptotic dynamics of the Leslie-Gower model (13) is well studied, see, for instance, Cushing et al. [10].

It can also be generalized to more than two species. In fact, for $k$ species interacting in an environment, the $k$-dimensional Leslie Gower competition model is given by

$$
\left\{\begin{aligned}
x_{1}(n+1)= & \frac{b_{1} x_{1}(n)}{1+\sum_{i=1}^{k} c_{1 i} x_{i}(n)} \\
x_{2}(n+1)= & \frac{b_{2} x_{2}(n)}{1+\sum_{i=1}^{k} c_{2 i} x_{i}(n)} . \\
& \vdots \\
x_{k}(n+1)= & \frac{b_{k} x_{k}(n)}{1+\sum_{i=1}^{k} c_{k i} x_{i}(n)}
\end{aligned}\right.
$$

It is assumed that the parameters are positive and the inherent growth rate of each species is such that in the absence of competition each species will growth to its individual carrying capacity. Notice that if $b_{i} \leq 1$, for any $i=1,2, \ldots, k$, then species $x_{i}$ goes to extinction, i.e. $x_{i}(n) \rightarrow 0$ as $n \rightarrow \infty$. Since our main assumption is that our system does not go to extinction, we will only consider the system (14) when $b_{i}>1$, for all $i=1,2, \ldots, k$. We also observe that the origin is a fixed point.

We shall now focus our global stability analyses for the planar and the three dimensional Leslie-Gower model.

\subsubsection{Planar Leslie-Gower model}

We can represent the planar Leslie-Gower system (13) by the map $F: \mathbb{R}_{+}^{2} \rightarrow \mathbb{R}_{+}^{2}$ given by

$$
F\left(x_{1}, x_{2}\right)=\left(\frac{b_{1} x_{1}}{1+c_{11} x_{1}+c_{12} x_{2}}, \frac{b_{2} x_{2}}{1+c_{21} x_{1}+c_{22} x_{2}}\right) .
$$

Under some restrictions on the parameters, the model has at most one interior fixed point and the dynamics is similar to the classical Lotka-Volterra competition system of ordinary differential equations [10].

Our goal is to show that the planar Leslie-Gower satisfies the assumptions of our main result and thus we will conclude that the unique interior fixed point is globally 
asymptotically stable. First, we compute the Jacobian matrix of $F$ which is given by

$$
J F\left(x_{1}, x_{2}\right)=\left(\begin{array}{cc}
\frac{b_{1}\left(1+c_{12} x_{2}\right)}{\left(1+c_{11} x_{1}+c_{12} x_{2}\right)^{2}} & -\frac{c_{12} b_{1} x_{1}}{\left(1+c_{11} x_{1}+c_{12} x_{2}\right)^{2}} \\
-\frac{c_{21} b_{2} x_{2}}{\left(1+c_{21} x_{1}+c_{22} x_{2}\right)^{2}} & \frac{b_{2}\left(1+c_{21} x_{1}\right)}{\left(1+c_{21} x_{1}+c_{22} x_{2}\right)^{2}}
\end{array}\right) .
$$

It is straightforward to see that the origin is an unstable fixed point of $F$ since the eigenvalues of $J F(0)$ are $b_{i}>1$ for $i=1,2$. Our next objective is to show that the map $F$ satisfy (H1). We will do so by computing the determinant of the Jacobian matrix, which is given by

$$
\operatorname{det} J F\left(x_{1}, x_{2}\right)=\frac{b_{1} b_{2}\left(c_{21} x_{1}+c_{12} x_{2}+1\right)}{\left(c_{11} x_{1}+c_{12} x_{2}+1\right)^{2}\left(c_{21} x_{1}+c_{22} x_{2}+1\right)^{2}}>0 .
$$

Thus, $F$ is orientation preserving and for all $\mathbf{x} \in \mathbb{R}_{+}^{2}, F$ satisfies (H1) by taking $R=\mathbb{R}_{+}^{2}$. Next, we consider (H2) and check that $F$ is a monotone map. In fact, by Lemma 4.1 we compute the cofactors of $J F$ and obtain

$$
\begin{aligned}
& C_{11}\left(J F\left(x_{1}, x_{2}\right)\right)=\frac{b_{2}\left(c_{21} x_{1}+1\right)}{\left(c_{21} x_{1}+c_{22} x_{2}+1\right)^{2}}, \\
& C_{12}\left(J F\left(x_{1}, x_{2}\right)\right)=\frac{b_{2} c_{21} x_{2}}{\left(c_{21} x_{1}+c_{22} x_{2}+1\right)^{2}}, \\
& C_{21}\left(J F\left(x_{1}, x_{2}\right)\right)=\frac{b_{1} c_{12} x_{1}}{\left(c_{11} x_{1}+c_{12} x_{2}+1\right)^{2}}, \\
& C_{22}\left(J F\left(x_{1}, x_{2}\right)\right)=\frac{b_{1}\left(c_{12} x_{2}+1\right)}{\left(c_{11} x_{1}+c_{12} x_{2}+1\right)^{2}} .
\end{aligned}
$$

Since all four cofactors are positive, we conclude that map $F$ is monotone and (H2) is satisfied.

We now consider hypotheses (H3), that is, the stability analysis of the fixed points in the coordinate subspaces. First, it is well-known that $x^{*}=\frac{b-1}{c}$ is a global asymptotically stable fixed point of the one-dimensional Leslie-Gower map $f(x)=\frac{b x}{1+c x}$ whenever $b>1$. For the planar Leslie-Gower map, $F$ has four fixed points. The origin, which is a unstable fixed point, two exclusions fixed points on the axis $E_{1}=\left(\frac{b_{1}-1}{c_{11}}, 0\right)$ and $E_{2}=\left(0, \frac{b_{2}-1}{c_{22}}\right)$ and, possibly, a coexistence fixed point (positive fixed point in the interior of the first quadrant) given by

$$
E^{*}=\left(\frac{\left(b_{1}-1\right) c_{22}-\left(b_{2}-1\right) c_{12}}{c_{11} c_{22}-c_{12} c_{21}}, \frac{\left(b_{2}-1\right) c_{11}-\left(b_{1}-1\right) c_{21}}{c_{11} c_{22}-c_{12} c_{21}}\right)
$$

Since our main objective is to study the global stability of $E^{*}$, we will have two scenarios in order to guarantee that $E^{*}$ exists and it is a positive fixed point (Figure 7). 


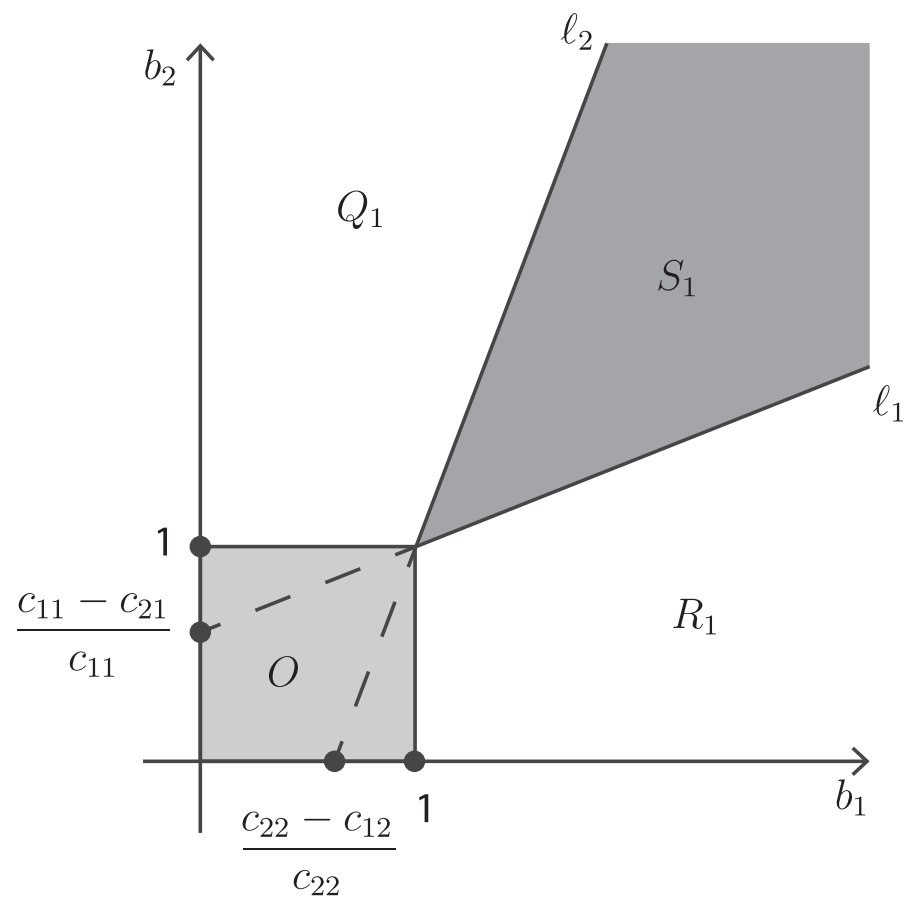

Figure 7. Region $S_{1}$ in the parameter space $b_{1} O b_{2}$ correspond to set where conditions (15) are satisfied. If $\left(b_{1}, b_{2}\right) \in S_{1}$ the positive fixed point $E^{*}$ is globally asymptotically stable (GAS). If $\left(b_{1}, b_{2}\right) \in O$ the origin is GAS. If $\left(b_{1}, b_{2}\right) \in R_{1}$ the exclusion fixed point $E_{1}$ is GAS while if $\left(b_{1}, b_{2}\right) \in Q_{1}$ the exclusion fixed point $E_{2}$ is GAS. Lines $I_{1}$ and $I_{2}$ is where we have equality in (15).

Case (i): Suppose $c_{11} c_{22}>c_{12} c_{21},\left(b_{1}-1\right) c_{22}>\left(b_{2}-1\right) c_{12}$ and $\left(b_{2}-1\right) c_{11}>$ $\left(b_{1}-1\right) c_{21}$, or equivalently,

$$
\frac{c_{12}}{c_{22}}<\frac{b_{1}-1}{b_{2}-1}<\frac{c_{11}}{c_{21}} .
$$

A straightforward manipulation of the inequalities in (15) yields

$$
\frac{b_{2} c_{11}}{\left(b_{1}-1\right) c_{21}+c_{11}}>1 \text { and } \frac{b_{1} c_{22}}{\left(b_{2}-1\right) c_{12}+c_{22}}>1 \text {. }
$$

Next, the eigenvalues of $J F$, at the fixed points $E_{1}$ and $E_{2}$ are given by

$$
\sigma\left(J F\left(E_{1}\right)\right)=\left\{\frac{b_{2} c_{11}}{\left(b_{1}-1\right) c_{21}+c_{11}}, \frac{1}{b_{1}}\right\}
$$

and

$$
\sigma\left(J F\left(E_{2}\right)\right)=\left\{\frac{b_{1} c_{22}}{\left(b_{2}-1\right) c_{12}+c_{22}}, \frac{1}{b_{2}}\right\} .
$$

From (16) and the fact that $\frac{1}{b_{i}}<1$, for $i=1,2$, it follows that $E_{1}$ and $E_{2}$ are saddle fixed points. Thus, hypothesis (H3) is satisfied. 
Case (ii) Suppose $c_{11} c_{22}<c_{12} c_{21},\left(b_{1}-1\right) c_{22}<\left(b_{2}-1\right) c_{12}$ and $\left(b_{2}-1\right) c_{11}<$ $\left(b_{1}-1\right) c_{21}$, or equivalently,

$$
\frac{c_{11}}{c_{21}}<\frac{b_{1}-1}{b_{2}-1}<\frac{c_{12}}{c_{22}} .
$$

In this scenario, it is an easy computation to verify that $E_{1}$ and $E_{2}$ are both locally asymptotically stable and $E^{*}$ is saddle. This is a phenomenon known in population dynamics as the competitive exclusion principle, also referred to as Gause Law of competitive exclusion, see [14]. It states that two species that compete for the exact same resources cannot stably coexist. In other words the dynamics of the system depends on initial conditions. There exists a separatrix in the first quadrant where the orbit goes either to $x$-axis or $y$-axis depending on the location of the initial condition. Hence only one species will survive. Since this scenario does not fit the objectives of the paper, that is, no extinction of any species, we shall not consider it.

Finally, as we consider the Leslie-Gower model with parameters satisfying condition (15) in case (i), we find that (H1)-(H4) are satisfied and there is a unique positive fixed point $E^{*}$ of $F$. Thus by Theorem 3.2, it follows that the coexistence fixed point is globally asymptotically stable in the interior of $\mathbb{R}_{+}^{2}$.

\subsubsection{Three Dimensional Leslie-Gower model}

We now consider the three dimensional Leslie-Gower model, $F: \mathbb{R}_{+}^{3} \rightarrow \mathbb{R}_{+}^{3}$ given by

$$
F\left(x_{1}, x_{2}, x_{3}\right)=\left(\frac{b_{1} x_{1}}{1+\sum_{i=1}^{3} c_{1 i} x_{i}}, \frac{b_{2} x_{2}}{1+\sum_{i=1}^{3} c_{2 i} x_{i}}, \frac{b_{3} x_{3}}{1+\sum_{i=1}^{3} c_{3 i} x_{i}}\right) .
$$

It is a straightforward, but long computation to show that $F$ is orientation preserving as $\operatorname{det} J F>0$ and the cofactors of $J F$ are positive. This computation is given in the Appendix 1 . Thus, we can immediately see that $F$ trivially satisfies $(\mathrm{H} 1)$ by taking the monotone region to be $R=\mathbb{R}_{+}^{3}$ and that $F$ is monotone, that is, it satisfies (H2).

It remains to determine if $F$ satisfy (H3). In this direction, we find that there are eight fixed points of $F$ as follows: the origin, three fixed points on each axis, three fixed points on each plane, and a coexistence fixed point $E^{*}$. For each coordinate plane, from Section 5.1.1 it follows that the coexistence fixed point of the restriction of $F$ to each planar subspace is globally asymptotically stable. In addition, we assume that the three fixed points on each plane given below have positive coordinates, except for one that is clearly zero. The fixed points are

$$
\begin{aligned}
& E_{12}=\left(\frac{\left(b_{1}-1\right) c_{22}-\left(b_{2}-1\right) c_{12}}{c_{11} c_{22}-c_{12} c_{21}}, \frac{\left(b_{2}-1\right) c_{11}-\left(b_{1}-1\right) c_{21}}{c_{11} c_{22}-c_{12} c_{21}}, 0\right) \\
& E_{13}=\left(\frac{\left(b_{1}-1\right) c_{33}-\left(b_{3}-1\right) c_{13}}{c_{11} c_{33}-c_{13} c_{31}}, 0, \frac{\left(b_{3}-1\right) c_{11}-\left(b_{1}-1\right) c_{31}}{c_{11} c_{33}-c_{13} c_{31}}\right)
\end{aligned}
$$

and

$$
E_{23}=\left(0, \frac{\left(b_{2}-1\right) c_{33}-\left(b_{3}-1\right) c_{23}}{c_{22} c_{33}-c_{23} c_{32}}, \frac{\left(b_{3}-1\right) c_{22}-\left(b_{2}-1\right) c_{32}}{c_{22} c_{33}-c_{23} c_{32}}\right)
$$


The coordinates of the coexistence fixed point $E^{*}$ are given in the Appendix 1 since they include long expressions for each of the coordinates. Nevertheless, because each of the previous fixed points have positive coordinates, except for those that are clearly zero, we conclude that $E^{*}$ is a positive coexistence fixed point.

Next, we need to determine stability of the fixed points interior to each coordinate planes to show that hypotheses (H3) holds. This task requires consideration of all 12 parameters given in the Leslie-Gower model. Since our main goal is to provide a concrete illustration on how to apply our main ideas, instead of considering the general case, we focus our analyses in the totally symmetric case, i.e. $c_{i j}=c, i \neq j$ and $c_{i i}=1$ and $b=b_{i}$, for $i, j=1,2,3$.

By symmetry let us consider the fixed point $E_{12}$ which we will show it is a saddle. We have two possible scenarios: either $c<1$ or $c \geq 1$. In the last case where $c \geq 1$, the system depends on initial conditions and the behaviour will be similar as we described in case (ii) for the planar system. Hence, this scenario does not fit the assumptions and will not be considered. Therefore, we assume $c<1$.

The eigenvalues of $J F\left(E_{12}\right)$ are given by

$$
\sigma\left(J F\left(E_{12}\right)\right)=\left\{\frac{1}{b}, \frac{2 b c-c+1}{b c+b}, \frac{b(c+1)}{(2 b-1) c+1}\right\} .
$$

Since $c<1$, a simple computation shows that $\frac{b(c+1)}{(2 b-1) c+1}>1$. By the fact that $b>1$ we have that $\frac{1}{b}<1$ and $\frac{2 b c-c+1}{b c+b}<1$. This implies that $E_{12}$ is a saddle. A similar analyses shows that this is the case for $E_{13}$ and $E_{23}$ as well.

Consequently, hypothesis (H3) is verified whenever $b=b_{i}, c_{i i}=1$ and $c_{i j}=c<1$, $i \neq j$ for all $i, j=1,2,3$.

Since $(\mathrm{H} 1)-(\mathrm{H} 4)$ is satisfied and there is a unique positive fixed point of $F$, it follows by Theorem 2.4 that the coexistence fixed point $E^{*}$ is globally asymptotically stable.

\subsection{Ricker competition model}

The planar Ricker competition model is given by the following autonomous difference equation:

$$
\left\{\begin{array}{l}
x_{1}(n+1)=x_{1}(n) \exp \left(r_{1}-c_{11} x_{1}(n)-c_{12} x_{2}(n)\right) \\
x_{2}(n+1)=x_{2}(n) \exp \left(r_{2}-c_{21} x_{1}(n)-c_{22} x_{2}(n)\right)
\end{array}, n \in \mathbb{Z}^{+} .\right.
$$

Here $x_{1}(n)$ and $x_{2}(n)$ represents the population sizes of two species at time unit $n$. The parameters $r_{1}$ and $r_{2}$ are the inherent exponential growth rates at low densities and $c_{i, j}, i, j=1,2$, are the competition intensity coefficients measuring the effects of intraspecific competition and inter-specific competition. More precisely, $c_{11}$ and $c_{22}$ are the intra-specific competition parameters while $c_{12}$ and $c_{21}$ are the inter-specific competition parameters. Notice that, these six parameters are assumed to be positive. The dynamics of the Ricker competition model has been well studied, see for instance, $[1,10,26]$.

The Ricker competition model can also be generalized to more than two species. In fact, for $k$ species interacting in an environment, the $k$-dimensional Ricker competition model 
is given by

$$
\left\{\begin{array}{c}
x_{1}(n+1)=x_{1}(n) \exp \left(r_{1}-\sum_{i=1}^{k} c_{1 i} x_{i}(n)\right) \\
x_{2}(n+1)=x_{2}(n) \exp \left(r_{2}-\sum_{i=1}^{k} c_{2 i} x_{i}(n)\right) \\
\vdots \\
x_{k}(n+1)=x_{k}(n) \exp \left(r_{k}-\sum_{i=1}^{k} c_{k i} x_{i}(n)\right)
\end{array} .\right.
$$

We shall now focus our attention to establish global stability for the planar and the three dimensional Ricker competition model.

\subsubsection{Planar Ricker competition model}

When considering the planar Ricker competition model, we can scale the state variables against the inherent carrying capacities to reduce the number of parameters and represent (18) by the map $F: \mathbb{R}_{+}^{2} \rightarrow \mathbb{R}_{+}^{2}$ given by

$$
F\left(x_{1}, x_{2}\right)=\left(x_{1} e^{r_{1}-x_{1}-a x_{2}}, x_{2} e^{r_{2}-b x_{1}-x_{2}}\right) .
$$

Since we interested in the analyses of a system that does not go to extinction and has a unique positive coexistence fixed point, a simple computation establishes that $a r_{2}<r_{1}$, $b r_{1}<r_{2}$, and $a b<1$, see [6] for details.

It is a well-known result from Smith [32, Proposition 6.1], that when $0 \leq r_{1}, r_{2} \leq 1$, local stability of the unique positive coexistence fixed point implies global stability. As mentioned in the beginning of this section, the crucial step in the proof of this theorem makes reference to an unpublished work. Recent work by the authors in [6] using singularity theory, can easily show global stability of the monotone planar Ricker competition model. We shall take the opportunity to illustrate how to apply our ideas and verify our conditions to have a recorded version of the proof of this important result.

Let us compute the Jacobian matrix of $F$ which is given by

$$
J F\left(x_{1}, x_{2}\right)=\left(\begin{array}{cc}
\left(1-x_{1}\right) e^{r_{1}-x_{1}-a x_{2}} & -a x_{2} e^{r_{1}-x_{1}-a x_{2}} \\
-b x_{2} e^{r_{2}-b x_{1}-x_{2}} & \left(1-x_{2}\right) e^{r_{2}-b x_{1}-x_{2}}
\end{array}\right) .
$$

It is straightforward to see that the origin is an unstable fixed point of $F$ since the eigenvalues of $J F(0)$ are $e^{r_{i}}>1$ for $i=1,2$. Our next objective is find the monotone region $R$ that satisfy ( $\mathrm{H} 1)$ and $(\mathrm{H} 2)$.

First, the determinant of $J F$ is given by

$$
\operatorname{det} J F\left(x_{1}, x_{2}\right)=e^{r_{1}+r_{2}-x_{1}-b x_{1}-x_{2}-a x_{2}}\left(1-x_{1}-x_{2}+x_{1} x_{2}-a b x_{1} x_{2}\right) .
$$

The set where det $J F\left(x_{1}, x_{2}\right)=0$ is called the critical curve $L C_{-1}$ and it is given by

$$
L C_{-1}=\left\{\left(x_{1}, x_{2}\right) \in \mathbb{R}_{+}^{2}: x_{2}=\frac{1-x_{1}}{1-(1-a b) x_{1}}, x_{1} \neq \frac{1}{1-a b}\right\} .
$$

There exists a region $\mathcal{R}_{1}$ containing the origin so that whenever $\left(x_{1}, x_{2}\right) \in \mathcal{R}_{1}$, $\operatorname{det} J F\left(x_{1}, x_{2}\right)>0$. Indeed, $\mathcal{R}_{1}$ is the region containing the origin bounded by the left component of $L C_{-1}$, denoted by $L C_{-1}^{1}$ (see [6] for details). We claim that $\mathcal{R}_{1}$ is a monotone region we seek. 
Indeed, $\left.F\right|_{\mathcal{R}_{1}}$ is orientation preserving and by [6, Theorem 4.7], we have that $F\left(\mathcal{R}_{1}\right)=$ $F\left(\mathbb{R}_{+}^{2}\right)$. Finally, to check that $\partial \mathcal{R}_{1}=L C_{-1}^{1}$ is a monotone curve (according to Definition 2.1), we can simply consider the function $\varphi:[0,1] \rightarrow \mathbb{R}$ given by $\varphi(t)=\frac{1-t}{1-(1-a b) t}$, and observe that $\varphi^{\prime}(t)=-\frac{a b}{(a b t-t+1)^{2}}<0$. Thus (H1) is satisfied with $R=\mathcal{R}_{1}$.

Furthermore, we remark that $\mathcal{R}_{1}$ is a convex region. Indeed, computing the second derivative of the parameterization of $h$ shows that

$$
\varphi^{\prime \prime}(t)=-\frac{2 a b(1-a b)}{(1-(1-a b) t)^{3}}<0 .
$$

Next, we consider the cofactors of $J F$ which are given by:

$$
\begin{aligned}
& C_{11}\left(J F\left(x_{1}, x_{2}\right)\right)=e^{K}\left(1-x_{2}\right), \\
& C_{12}\left(J F\left(x_{1}, x_{2}\right)\right)=e^{K} b x_{2}, \\
& C_{21}\left(J F\left(x_{1}, x_{2}\right)\right)=e^{K} a x_{1}, \\
& C_{22}\left(J F\left(x_{1}, x_{2}\right)\right)=e^{K}\left(1-x_{1}\right),
\end{aligned}
$$

where $K=r_{1}+r_{2}-x_{1}-b x_{1}-x_{2}-a x_{2}$. Since all four cofactors are positive in $\mathcal{R}_{1}$ as $x_{1}, x_{2}<1$, we conclude from Lemma 4.1 the map $F$ is monotone and (H2) is satisfied.

We now consider hypotheses (H3). It is well-known [13] that $x^{*}=r$ is a global asymptotically stable fixed point of the one dimensional Ricker map $f(x)=x e^{r-x}$ whenever $0<r \leq 2$. The planar Ricker competition model has four fixed points: the origin, which is an unstable fixed point, two exclusions fixed points on the axis $E_{1}=\left(r_{1}, 0\right)$ and $E_{2}=\left(0, r_{2}\right)$ and, possibly, a coexistence fixed point given by

$$
E^{*}=\left(\frac{r_{1}-a r_{2}}{1-a b}, \frac{r_{2}-b r_{1}}{1-a b}\right) \text {. }
$$

When $a b>1$ we have the exclusion principle, see [26] for details, and this shall not be considered. As mentioned before we will consider the case $a b<1, r_{2}>b r_{1}$, and $r_{1}>a r_{2}$ which ensures the existence of a unique positive coexistence fixed point. In addition, we are considering the inherent growth rates to be less than 1 , i.e. $r_{i}<1, i=1,2$.

Next, we compute the eigenvalues of $J F$ at the fixed points $E_{1}$ and $E_{2}$ which are given by

$$
\sigma\left(J F\left(E_{1}\right)\right)=\left\{e^{r_{2}-b r_{1}}, 1-r_{1}\right\} \text { and } \sigma\left(J F\left(E_{2}\right)\right)=\left\{e^{r_{1}-a r_{2}}, 1-r_{2}\right\} .
$$

Due the restrictions on the parameters it follows that $E_{1}$ and $E_{2}$ are saddle fixed points on the axis. Thus, hypothesis (H3) is satisfied.

In summary, as we consider the Ricker competition model with parameters satisfying $a b<1, b r_{1}<r_{2}<1$ and $a r_{2}<r_{1}<1$ we have that hypotheses (H1)-(H4) are satisfied and there is a unique coexistence fixed $E^{*}$ of $F$. It now follows from Theorem 2.4 that the coexistence fixed point $E^{*}$ is globally asymptotically stable in the interior of $\mathbb{R}_{+}^{2}$. 


\subsubsection{Three dimensional Ricker competition model}

We now focus on the three dimensional Ricker competition model where the map $F$ : $\mathbb{R}_{+}^{3} \rightarrow \mathbb{R}_{+}^{3}$ is given by

$$
F\left(x_{1}, x_{2}, x_{3}\right)=\left(x_{1} e^{r_{1}-x_{1}-a_{12} x_{2}-a_{13} x_{3}}, x_{2} e^{r_{2}-a_{21} x_{1}-x_{2}-a_{23} x_{3}}, x_{3} e^{r_{3}-a_{31} x_{1}-a_{32} x_{2}-x_{3}}\right) .
$$

Our objective is to find a region of parameters of the three dimensional Ricker competition map where the assumptions $(\mathrm{H} 1)-(\mathrm{H} 3)$ are satisfied.

We will begin as we did in the planar case. First, we shall restrict ourselves to the case where the interior positive fixed point exists. The computation of $E^{*}$ is straightforward, but involve long expressions. Thus we include the details of the restrictions of the conditions on the parameters, to be denoted by (B2), in Appendix 2.

We will show that the three dimensional analogue of $\mathcal{R}_{1}$ is also a monotone region. Then, in order to show it satisfies (H1), we need the analogue of [6, Theorem 4.7]. We conjecture that the same characterization of the local structure of planar singularities as it is done by Whitney [33] could be obtained in higher dimensions, but we are not aware of such results. Nevertheless, we can still establish a condition on the parameters of the Ricker competition model that will ensure that the image will be contained in $\mathcal{R}_{1}$ and hence satisfy ( $\mathrm{H} 1)$.

Let us begin by computing the Jacobian matrix of $F$ and its determinant to find the set of singularities of the Ricker map. A computation establishes that

$$
J F:=J F\left(x_{1}, x_{2}, x_{3}\right)=\left(\begin{array}{ccc}
\left(1-x_{1}\right) e^{K_{1}} & -a_{12} x_{1} e^{K_{1}} & -a_{13} x_{1} e^{K_{1}} \\
-a_{21} x_{2} e^{K_{2}} & \left(1-x_{2}\right) e^{K_{2}} & -a_{23} x_{2} e^{K_{2}} \\
-a_{31} x_{3} e^{K_{3}} & -a_{32} x_{3} e^{K_{3}} & \left(1-x_{3}\right) e^{K_{3}}
\end{array}\right),
$$

where

$$
\begin{aligned}
& K_{1}=r_{1}-x_{1}-a_{12} x_{2}-a_{13} x_{3}, \\
& K_{2}=r_{2}-a_{21} x_{1}-x_{2}-a_{23} x_{3},
\end{aligned}
$$

and

$$
K_{3}=r_{3}-a_{31} x_{1}-a_{32} x_{2}-x_{3} .
$$

Next, we can compute its determinant and obtain det $J F=e^{K_{1}+K_{2}+K_{3}} \Delta$, where

$$
\begin{aligned}
\Delta= & 1-\left(x_{1}+x_{2}+x_{3}\right)+\left(1-a_{12} a_{21}\right) x_{1} x_{2}+\left(1-a_{13} a_{31}\right) x_{1} x_{3}+\left(1-a_{23} a_{32}\right) x_{2} x_{3} \\
& -\left(1-a_{12} a_{21}-a_{13} a_{31}-a_{23} a_{32}+a_{12} a_{23} a_{31}+a_{13} a_{21} a_{32}\right) x_{1} x_{2} x_{3} .
\end{aligned}
$$

We are interested in the set $L C_{-1}=\left\{\left(x_{1}, x_{2}, x_{3}\right) \in \mathbb{R}_{+}^{3}\right.$ : det $\left.J F=0\right\}$. In particular, in the component of $L C_{-1}$, denoted by $L C_{-1}^{1}$ which bounds a region $\mathcal{R}_{1}$ containing the origin and where det $J F>0$. Solving $\Delta=0$ for $x_{3}$ using (25), we have that $L C_{-1}^{1}$ is the graph of a function $\Phi: \mathcal{R}_{x_{1} x_{2}} \rightarrow \mathbb{R}$ where $\mathcal{R}_{x_{1} x_{2}}$ is the region $\mathcal{R}_{1}$ restricted to the $x_{1} x_{2}$-plane.

Now, we can establish that $\mathcal{R}_{1}$ is a monotone region by simply computing the normal vector at each point in $\partial \mathcal{R}_{1}=L C_{-1}^{1}$. Since $L C_{-1}^{1}$ is a graph of the function $\Phi$, we have that $\eta=\left(-\partial_{x_{1}} \Phi,-\partial_{x_{2}} \Phi, 1\right)$. Because this is a long computation, we have included the details in Appendix 2. Indeed, (B4) shows that $\partial_{x_{1}} \Phi<0$ and $\partial_{x_{2}} \Phi<0$. Hence, we conclude that $\eta>0$ and $\mathcal{R}_{1}$ is a monotone region. 
In order to establish (H1) we need to show that the image of any point in $\mathbb{R}_{+}^{3}$ will eventually be contained in $\mathcal{R}_{1}$. First, denote $F=\left(f_{1}, f_{2}, f_{3}\right)$, and for $i=1,2,3$, we define the isocline $S_{i}=\left\{\left(x_{1}, x_{2}, x_{3}\right) \in \mathbb{R}_{+}^{3}: f_{i}\left(x_{1}, x_{2}, x_{3}\right)=x_{i}\right\}$, i.e. we have the following sets

$$
\begin{aligned}
& S_{1}=\left\{(x, y, z) \in \mathbb{R}_{+}^{3}: r_{1}-x-a_{12} y-a_{13} z=0\right\}, \\
& S_{2}=\left\{(x, y, z) \in \mathbb{R}_{+}^{3}: r_{2}-a_{21} x-y-a_{23} z=0\right\}, \\
& S_{3}=\left\{(x, y, z) \in \mathbb{R}_{+}^{3}: r_{3}-a_{31} x-a_{32} y-z=0\right\} .
\end{aligned}
$$

Let us also define $S_{i}^{+}=\left\{\left(x_{1}, x_{2}, x_{3}\right) \in \mathbb{R}_{+}^{3}: f_{i}\left(x_{1}, x_{2}, x_{3}\right)>x_{i}\right\}$ and $S_{i}^{-}=\left\{\left(x_{1}, x_{2}, x_{3}\right) \in\right.$ $\left.\mathbb{R}_{+}^{3}: f_{i}\left(x_{1}, x_{2}, x_{3}\right)<x_{i}\right\}$. Consider the following conditions

$\left(*_{1}\right)$ For each $i=1,2,3$, suppose $0 \leq r_{i} \leq a_{i j}$, for $i \neq j$.

$\left(*_{2}\right)$ The intra-specific parameters satisfy $a_{12} a_{23} a_{31}=a_{13} a_{32} a_{21}$.

We now prove the following result:

Lemma 5.1: Suppose for $i=1,2,3$ that $0 \leq r_{i} \leq 1$ and conditions $\left(*_{1}\right)$ and $\left(*_{2}\right)$ holds. Then, $L C_{-1}^{1} \subseteq S_{1}^{-} \cap S_{2}^{-} \cap S_{3}^{-}$.

Proof: We will show that $L C_{-1}^{1} \subseteq S_{i}^{-}$for each $i=1,2,3$. For instance, let us first consider the isocline $S_{1}$.

Observe that the point of intersection of $S_{1}$ with each of the coordinate axes is $\left(r_{1}, 0,0\right)$, $\left(0, \frac{r_{1}}{a_{12}}, 0\right),\left(0,0, \frac{r_{1}}{a_{13}}\right)$. The intersection of $L C_{-1}^{1}$ to each coordinate plane is a curve with negative second derivative as it was shown in (23). From condition $\left(*_{1}\right)$, we have $r_{1}, \frac{r_{1}}{a_{12}}, \frac{r_{1}}{a_{13}} \leq 1$ thus, for $i \neq j$, each endpoints of $S_{1} \cap H_{i j}$ is below $S_{1} \cap L C_{-1}^{1}$ and by convexity so is each segment $S_{1} \cap H_{i j}$ as depicted in Figure 8 .

Next, for each plane perpendicular to $x_{1}$ or $x_{2}$, the intersection of the plane and $S_{1}$ will be a segment that will be below $L C_{-1}^{1}$. Indeed, without loss of generality, for a fixed $t \in[0,1]$, let $H_{1}(t)$ denote the plane perpendicular to the coordinate axis $x_{1}$ containing $t$ and $H_{1}(t) \cap L C_{-1}^{1}$ be a curve denoted by $\gamma_{1}$. The concavity of $\gamma_{1}$ is determined by $\partial_{x_{2} x_{2}} \Phi$ and by (B5) in Appendix 2, we have that $\partial_{x_{2} x_{2}} \Phi<0$.

Therefore, as we restrict ourselves to the plane $H_{1}(t)$, the curve $\gamma_{1}$ bounds the endpoints of $H_{1}(t) \cap S_{1}$. By the concavity of $\gamma_{1}$, we have that $\gamma_{1}$ actually bounds the segment $H_{1}(t) \cap S_{1}$ and hence $L C_{-1}^{1} \subseteq S_{1}^{-}$. The argument is analogous for each $i$, hence, $L C_{-1}^{1} \subseteq S_{1}^{-} \cap S_{2}^{-} \cap$ $S_{3}^{-}$.

The main consequence of Lemma 5.1 is that under condition $\left(*_{1}\right)$, the image of any point $\mathbf{x}>0$ outside $\mathcal{R}_{1}$, will have all of the coordinates of $F(\mathbf{x})$ smaller than the coordinates $\mathbf{x}$. This means that $F$ is componentwise decreasing in $S_{1}^{-} \cap S_{2}^{-} \cap S_{3}^{-}$. Therefore, there must be $m \in \mathbb{N}$ such that $F^{m}(\mathbf{x}) \in \mathcal{R}_{1}$, that is, the orbit of $\mathbf{x}$ will eventually be in $\mathcal{R}_{1}$. This shows that under conditions $\left(*_{1}\right)$ and $\left(*_{2}\right)$, the region $\mathcal{R}_{1}$ is a monotone region that satisfies condition (H1). We remark that it is possible that after one iteration, the image of a point may not be inside $\mathcal{R}_{1}$. However, what it is actually important in condition (H1) is that eventually, the image of every point will be contained in $\mathcal{R}_{1}$.

Now, we shall determine conditions on the parameters that will ensure that the Ricker competition map is monotone and hence satisfies $(\mathrm{H} 2)$. Consider the following condition

$\left(*_{3}\right)$ For each $i=1,2,3$, suppose $a_{i j}+1<e^{1-r_{i}}$, for $i \neq j$. 


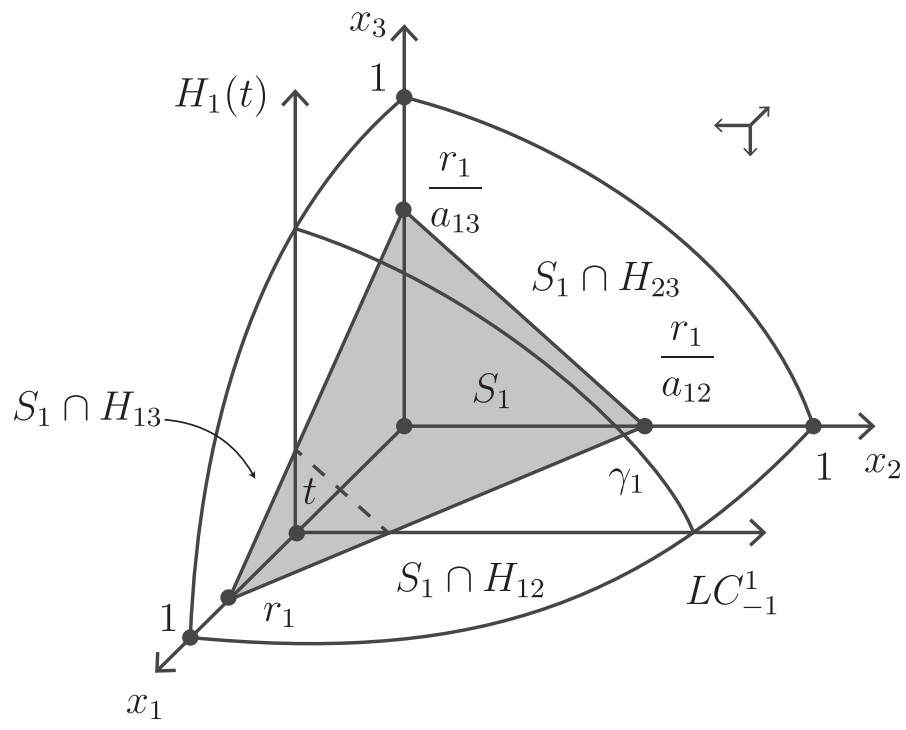

Figure 8. The isocline $S_{1}$, where $f_{1}\left(x_{1}, x_{2}, x_{3}\right)=x_{1}$, is inside the positive cone and bounded by segments that are inside $\mathcal{R}_{1}$. Each plane perpendicular to the coordinate axis intersects $L C_{-1}^{1}$ in a curve $\gamma_{i}$ that bounds the segments in $S_{1}$.

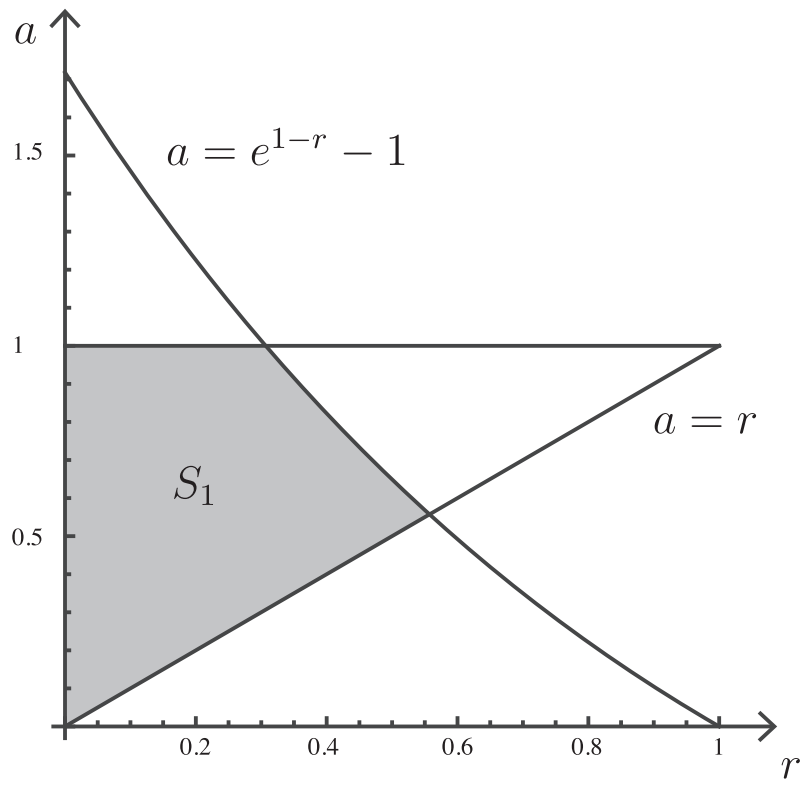

Figure 9. The region $S_{1}$, in the parameter space $r O a$, corresponds to set where conditions $\left(*_{1}\right),\left(*_{2}\right)$, and $\left(*_{3}\right)$ are satisfied, i.e. $0<r<a<1$ and $a<e^{1-r}-1$. If $(r, a) \in S_{1}$ the positive fixed point $E^{*}$ is globally asymptotically stable (GAS) with respect to the interior of the positive orthant. 
Lemma 5.2: Suppose for $i=1,2,3$ that $0 \leq r_{i} \leq 1$ and condition $\left(*_{3}\right)$ holds. Then, the cofactors of JF are positive and $F$ is monotone.

Proof: From the expression of $J F$ in (24), we can write $J F=e^{K} M$, where $M$ is the matrix given below. Thus it suffices to show that the cofactors of $M$ are positive. Indeed,

$$
M=\left(\begin{array}{ccc}
1-x_{1} & -a_{12} x_{1} & -a_{13} x_{1} \\
-a_{21} x_{2} & 1-x_{2} & -a_{23} x_{2} \\
-a_{31} x_{3} & -a_{32} x_{3} & 1-x_{3}
\end{array}\right)
$$

and the cofactors of $M$ are given by

$$
\begin{aligned}
C_{j k} & =x_{k}\left(a_{k j}\left(1-x_{i}\right)+a_{k i} a_{i j} x_{i}\right), \\
C_{i i} & =\left(1-x_{j}\right)\left(1-x_{k}\right)-a_{j k} a_{k j} x_{j} x_{k}
\end{aligned}
$$

where $\{i, j, k\}=\{1,2,3\}$. First, let us consider the nondiagonal cofactors $C_{j k}$ given in (27). Because we are considering $F$ restricted to $\mathcal{R}_{1}$ and we are only interested in the points in the interior of $\mathcal{R}_{1}$, we trivially have $0<x_{i} \leq 1$ and thus $C_{j k}>0$.

Next, for the diagonal cofactors $C_{i i}$ given in (28), we see that an algebraic manipulation together with $\left(*_{3}\right)$ shows that $C_{i i}>0$. Indeed, assume that $0<x_{j}, x_{k}$, then for each $j=1,2,3$, we have $x_{j} \leq e^{r_{j}-1}$, that is, $\frac{1}{x_{j}} \geq e^{1-r_{j}}$. Thus,

$$
\left(\frac{1}{x_{j}}-1\right) \geq e^{1-r_{j}}-1 \text { and }\left(\frac{1}{x_{k}}-1\right) \geq e^{1-r_{k}}-1
$$

From $\left(*_{2}\right), e^{1-r_{j}}-1>a_{j k}$ and $e^{1-r_{k}}-1>a_{k j}$, thus

$$
\left(\frac{1}{x_{j}}-1\right)\left(\frac{1}{x_{k}}-1\right) \geq\left(e^{1-r_{k}}-1\right)\left(e^{1-r_{j}}-1\right)>a_{j k} a_{k j}
$$

Multiplying (29) by $x_{j} x_{k}$ and simplifying, we conclude that $C_{i i}>0$.

It is now clear from Lemma 5.2 that if the parameters of the Ricker competition model satisfies Condition ( $*_{3}$ ), then $F$ satisfy (H2).

Now, it remains to determine under which conditions on the parameters, the map $F$ will satisfy (H3). In this direction, we see that there are eight fixed points of $F$ as follows: the origin, three fixed points on each axis, three fixed points on each plane, and a coexistence fixed point $E^{*}$. Since the model has nine parameters, the computations and analysis of local stability of each fixed point is very difficult. Since our goal is to highlight the process one must undergo to verify our hypotheses, we will focus on the symmetric case, i.e. $r_{i}=r$ and $a_{i j}=a$ for all $i$ and $j$.

The fixed points on each axis are given by $E_{1}=(r, 0,0), E_{2}=(0, r, 0)$, and $E_{3}=(0,0, r)$. Next, the three fixed points on each plane are

$$
E_{12}=\left(\frac{r}{1+a}, \frac{r}{1+a}, 0\right), \quad E_{13}=\left(\frac{r}{1+a}, 0, \frac{r}{1+a}\right) \quad \text { and } \quad E_{23}=\left(0, \frac{r}{1+a}, \frac{r}{1+a}\right) \text {. }
$$


Similarly to the Leslie-Gower model, in each coordinate plane, the restriction of $F$ behaves as the planar model. Hence, we assume that the parameters for each restriction satisfies the same conditions as the planar case, i.e. $r<1$ and $a<1$.

The coexistence fixed point $E^{*}$ is given by

$$
E^{*}=\left(\frac{r}{1+2 a}, \frac{r}{1+2 a}, \frac{r}{1+2 a}\right),
$$

which is clearly a positive fixed point.

Now, we turn our attention to the local stability of the fixed points in the coordinate planes. For instance, the eigenvalues of $E_{12}$ are

$$
\sigma\left(J F\left(E_{12}\right)\right)=\left\{e^{r \frac{1-a}{1+a}}, 1-r, \frac{a+1+(a-1) r}{a+1}\right\} .
$$

Since $\frac{1-a}{1+a}>0$, it follows that $e^{r \frac{1-a}{1+a}}>1$. Trivially, we verify that $1-r<1$. Next, from the condition that $a<1$, we have $a+1+r(a-1)<a+1$ and consequently $\frac{a+1+(a-1) r}{a+1}<1$. This implies that $E_{12}$ is a saddle fixed point, since $1-r<1$. Finally, similar conclusion can be taken in the case of $E_{13}$ and $E_{23}$ which are also saddle fixed points.

From Section 5.2.1 it follows that the coexistence fixed point of the restriction of $F$ to each planar subspace is globally asymptotically stable. Consequently, hypothesis (H3) is verified whenever $r<1$ and $a<1$.

This analyses shows that whenever $r_{i}=r, a_{i j}=a$, for all $i, j=1,2,3$ and $0<r<a<1$ and $a<e^{1-r}-1$, there is a unique positive fixed point $E^{*}$ given by (30) and the three dimensional Ricker competition model is globally asymptotically stable in the interior of $\mathbb{R}_{+}^{3}$. In Figure 9 we depict the set of parameters $r$ and $a$ that satisfy the global stability condition.

\section{Disclosure statement}

No potential conflict of interest was reported by the authors.

\section{Funding}

This work was partially supported by FCT/Portugal [project number PEst-OE/EEI/LA0009/2013].

\section{ORCID}

Rafael Luís (D) http://orcid.org/0000-0002-5991-9164

\section{References}

[1] A.S. Ackleh and P.L. Salceanu, Competitive exclusion and coexistence in an n-species Ricker model, J. Biol. Dyn. 9(sup1) (2015), pp. 321-331, pMID: 25783525.

[2] L. Assas, S. Elaydi, E. Kwessi, G. Livadiotis, and D. Ribble, Hierarchical competition models with Allee effects, J. Biol. Dyn. 9(sup1) (2015), pp. 32-44.

[3] S. Baigent, Geometry of carrying simplices of 3-species competitive Lotka-Volterra systems, Nonlinearity 26(4) (2013), pp. 1001-1029.

[4] E.C. Balreira, Foliations and global inversion, Comment. Math. Helv. 85(1) (2010), pp. 73-93. 
[5] E.C. Balreira, S. Elaydi, and R. Luís, Global dynamics of triangular maps, Nonlinear Anal. 104 (2014), pp. 75-83.

[6] E.C. Balreira, S. Elaydi, and R. Luís, Local stability implies global stability for the planar Ricker competition model, Discrete Contin. Dyn. Syst. Ser. B 19(2) (2014), pp. 323-351.

[7] S. Basu, A. Gabrielov, and N. Vorobjov, Monotone functions and maps, Rev. R. Acad. Cienc. Exactas Fís. Nat. Ser. A Math. RACSAM 107(1) (2013), pp. 5-33.

[8] S. Basu, A. Gabrielov, and N. Vorobjov, Semi-monotone sets, J. Eur. Math. Soc. (JEMS) 15(2) (2013), pp. 635-657.

[9] A. Cima, A. Gasull, and V. Mañosa, Basin of attraction of triangular maps with applications, J. Differ. Equ. Appl. 20(3) (2014), pp. 423-437.

[10] J. Cushing, S. Levarge, N. Chitnis, and S. Henson, Some discrete competition models and the competitive exclusion principle, J. Differ. Equ. Appl. 10(13-15) (2004), pp. 1139-1151.

[11] S. Elaydi, An Introduction to Difference Equations, 3rd ed., Springer, New York, 2005.

[12] S. Elaydi, Discrete Chaos: With Applications in Science and Engineering, 2nd ed., Chapman and Hall/CRC, Boca Raton, FL, 2008.

[13] S. Elaydi and R. Sacker, Basin of attraction of periodic orbits of maps on the real line, J. Differ. Equ. Appl. 10(10) (2004), pp. 881-888.

[14] G.F. Gause, The Struggle for Existence, The Williams \& Wilkins Company, Baltimore, MD, 1934.

[15] A. Hatcher, Algebraic Topology, Cambridge University Press, Cambridge, 2002.

[16] A. Ruiz-Herrera, Topological criteria of global attraction with applications in population dynamics, Nonlinearity 25(10) (2012), p. 2823-2842.

[17] A. Ruiz-Herrera, Exclusion and dominance in discrete population models via the carrying simplex, J. Differ. Equ. Appl. 19(1) (2013), pp. 96-113.

[18] M.W. Hirsch, On existence and uniqueness of the carrying simplex for competitive dynamical systems, J. Biol. Dyn. 2(2) (2008), pp. 169-179.

[19] S.B. Hsu, H.L. Smith, and P. Waltman, Competitive exclusion and coexistence for competitive systems on ordered Banach spaces, Trans. Amer. Math. Soc. 348(10) (1996), pp. 4083-4094.

[20] J. Hurt, Some stability theorems for ordinary difference equations, SIAM J. Numer. Anal. 4 (1967), pp. 582-596.

[21] J. Jiang, J. Mierczyński, and Y. Wang, Smoothness of the carrying simplex for discrete-time competitive dynamical systems: A characterization of neat embedding, J. Differ. Equ. 246(4) (2009), pp. 1623-1672.

[22] T. Konstantopoulos, A multilinear algebra proof of the Cauchy-Binet formula and a multilinear version of Parseval's identity, Linear Algebra Appl. 439(9) (2013), pp. 2651-2658.

[23] J. LaSalle, The Stability of Dynamical Systems, SIAM, Philadelphia, PA, 1976.

[24] J.M. Lee, Introduction to Smooth Manifolds, 2nd ed., Graduate Texts in Mathematics Vol. 218, Springer, New York, 2013.

[25] P. Leslie and J. Gower, The properties of a stochastic model for two competing species, Biometrica 45 (1958), pp. 316-330.

[26] R. Luís, S. Elaydi, and H. Oliveira, Stability of a Ricker-type competition model and the competitive exclusion principle, J. Biol. Dyn. 5(6) (2011), pp. 636-660.

[27] J. Quandt, On the Hartman-Grobman theorem for maps, J. Differ. Equ. 64(2) (1986), pp. 154164.

[28] C. Robinson, Dynamical Systems: Stability, Symbolic Dynamics, and Chaos, 2nd ed., CRC Press, Boca Raton, FL, 1999.

[29] W.E. Ricker, Stock and recruitment, J. Fish. Res. Board Can. 11(5) (1954), pp. 559-623.

[30] S. Smale, On the differential equations of species in competition, J. Math. Biol. 3(1) (1976), pp. $5-7$.

[31] H.L. Smith, Periodic competitive differential equations and the discrete dynamics of competitive maps, J. Differ. Equ. 64(2) (1986), pp. 165-194.

[32] H. Smith, Planar competitive and cooperative difference equations, J. Differ. Equ. Appl. 3(5-6) (1998), pp. 335-357. 
[33] $\mathrm{H}$. Whitney, On singularities of mappings of Euclidean spaces. Mappings of the plane into the plane, Ann. Math. 62(3) (1955), pp. 374-410.

\section{Appendix 1. Computations related to the 3D Leslie-Gower model}

The three dimensional Leslie-Gower model $F: \mathbb{R}_{+}^{3} \rightarrow \mathbb{R}_{+}^{3}$ is given by

$$
F\left(x_{1}, x_{2}, x_{3}\right)=\left(\frac{b_{1} x_{1}}{1+\sum_{i=1}^{3} c_{1 i} x_{i}}, \frac{b_{2} x_{2}}{1+\sum_{i=1}^{3} c_{2 i} x_{i}}, \frac{b_{3} x_{3}}{1+\sum_{i=1}^{3} c_{3 i} x_{i}}\right) .
$$

A computation of the Jacobian matrix shows that the matrix $J F:=J F\left(x_{1}, x_{2}, x_{3}\right)$ in this case is given by

$$
J F=\left(\begin{array}{ccc}
\frac{b_{1}\left(c_{12} x_{2}+c_{13} x_{3}+1\right)}{\left(c_{11} x_{1}+c_{12} x_{2}+c_{13} x_{3}+1\right)^{2}} & -\frac{b_{1} c_{12} x_{1}}{\left(c_{11} x_{1}+c_{12} x_{2}+c_{13} x_{3}+1\right)^{2}} & -\frac{b_{1} c_{13} x_{1}}{\left(c_{11} x_{1}+c_{12} x_{2}+c_{13} x_{3}+1\right)^{2}} \\
-\frac{b_{2} c_{21} x_{2}}{\left(c_{21} x_{1}+c_{22} x_{2}+c_{23} x_{3}+1\right)^{2}} & \frac{b_{2}\left(c_{21} x_{1}+c_{23} x_{3}+1\right)}{\left(c_{21} x_{1}+c_{22} x_{2}+c_{23} x_{3}+1\right)^{2}} & -\frac{b_{2} c_{33} x_{2}}{\left(c_{21} x_{1}+c_{22} x_{2}+c_{23} x_{3}+1\right)^{2}} \\
-\frac{b_{3} c_{31} x_{3}}{\left(c_{31} x_{1}+c_{32} x_{2}+c_{33} x_{3}+1\right)^{2}} & -\frac{b_{3} c_{32} x_{3}}{\left(c_{31} x_{1}+c_{32} x_{2}+c_{33} x_{3}+1\right)^{2}} & \frac{b_{3}\left(c_{31} 1 c_{1}+c_{32} x_{2}+1\right)}{\left(c_{31} x_{1}+c_{32} x_{2}+c_{33} x_{3}+1\right)^{2}}
\end{array}\right) .
$$

Next, we compute the determinant of $J F$ and find that it is given by

$$
\begin{array}{r}
\frac{b_{1} b_{2} b_{3}\left(c_{21} x_{1}\left(c_{31} x_{1}+c_{32} x_{2}+c_{13} x_{3}+1\right)+c_{31} x_{1}\left(c_{12} x_{2}+c_{23} x_{3}+1\right)\right)}{\prod_{i=1}^{3}\left(1+\sum_{j=1}^{3} c_{i j} x_{j}\right)^{2}} \\
+\frac{\left(c_{12} x_{2}+c_{13} x_{3}+1\right)\left(c_{32} x_{2}+c_{23} x_{3}+1\right)}{\prod_{i=1}^{3}\left(1+\sum_{j=1}^{3} c_{i j} x_{j}\right)^{2}}>0
\end{array}
$$

Since det $J F>0$, we can check that $J F^{-1}>0$ because all the cofactors of $J F$ are positive. Indeed, a computation shows that the cofactors are, for $\{i, j, k\}=\{1,2,3\}$,

$$
\begin{aligned}
C_{i i}(J F) & =\frac{b_{j} b_{k}\left(c_{j k} x_{k}\left(c_{k i} x_{i}+1\right)+c_{k j} x_{j}\left(c_{j i} x_{i}+1\right)+\left(c_{k i} x_{i}+1\right)\left(c_{j i} x_{i}+1\right)\right)}{\left(c_{j 1} x_{1}+c_{j 2} x_{2}+c_{j 3} x_{3}+1\right)^{2}\left(c_{k 1} x_{1}+c_{k 2} x_{2}+c_{k 3} x_{3}+1\right)^{2}} \\
C_{j k}(J F) & =\frac{b_{k} b_{i} x_{k}\left(c_{k j}\left(c_{i j} x_{j}+1\right)+c_{k i} c_{i j} x_{i}+c_{i k} c_{k j} x_{k}\right)}{\left(c_{j 1} x_{1}+c_{j 2} x_{2}+c_{j 3} x_{3}+1\right)^{2}\left(c_{k 1} x_{1}+c_{k 2} x_{2}+c_{k 3} x_{3}+1\right)^{2}}
\end{aligned}
$$

Next, a computation of the fixed points of $F$ shows that the origin is an extinction fixed point, and $E_{1}=\left(\frac{b_{1}-1}{c_{11}}, 0,0\right), E_{2}=\left(0, \frac{b_{2}-1}{c_{22}}, 0\right)$, and $E_{3}=\left(0,0, \frac{b_{3}-1}{c_{33}}\right)$ are exclusion fixed points. In addition, the map $F$ has the following fixed points in the coordinate planes

$$
\begin{aligned}
& E_{12}=\left(\frac{\left(b_{1}-1\right) c_{22}-\left(b_{2}-1\right) c_{12}}{c_{11} c_{22}-c_{12} c_{21}}, \frac{\left(b_{2}-1\right) c_{11}-\left(b_{1}-1\right) c_{21}}{c_{11} c_{22}-c_{12} c_{21}}, 0\right), \\
& E_{13}=\left(\frac{\left(b_{1}-1\right) c_{33}-\left(b_{3}-1\right) c_{13}}{c_{11} c_{33}-c_{13} c_{31}}, 0, \frac{\left(b_{3}-1\right) c_{11}-\left(b_{1}-1\right) c_{31}}{c_{11} c_{33}-c_{13} c_{31}}\right),
\end{aligned}
$$

and

$$
E_{23}=\left(0, \frac{\left(b_{2}-1\right) c_{33}-\left(b_{3}-1\right) c_{23}}{c_{22} c_{33}-c_{23} c_{32}}, \frac{\left(b_{3}-1\right) c_{22}-\left(b_{2}-1\right) c_{32}}{c_{22} c_{33}-c_{23} c_{32}}\right) .
$$

Recall that, in order to have the right dynamics, we have to assume conditions similar to Condition (15) for each one of these fixed points. 
The coordinates of the coexistence fixed point $E^{*}$ are given by

$$
\begin{aligned}
& \left(\frac{\left(b_{1}-1\right)\left(c_{23} c_{32}-c_{22} c_{33}\right)+\left(b_{2}-1\right)\left(c_{12} c_{33}-c_{13} c_{32}\right)+\left(b_{3}-1\right)\left(c_{13} c_{22}-c_{12} c_{23}\right)}{c_{13}\left(c_{22} c_{31}-c_{21} c_{32}\right)+c_{12}\left(c_{21} c_{33}-c_{23} c_{31}\right)+c_{11}\left(c_{23} c_{32}-c_{22} c_{33}\right)},\right. \\
& \frac{\left(b_{1}-1\right)\left(c_{21} c_{33}-c_{23} c_{31}\right)+\left(b_{2}-1\right)\left(c_{13} c_{31}-c_{11} c_{33}\right)+\left(b_{3}-1\right)\left(c_{11} c_{23}-c_{13} c_{21}\right)}{c_{13}\left(c_{22} c_{31}-c_{21} c_{32}\right)+c_{12}\left(c_{21} c_{33}-c_{23} c_{31}\right)+c_{11}\left(c_{23} c_{32}-c_{22} c_{33}\right)}, \\
& \left.\frac{\left(b_{1}-1\right)\left(c_{22} c_{31}-c_{21} c_{32}\right)+\left(b_{2}-1\right)\left(c_{11} c_{32}-c_{12} c_{31}\right)+\left(b_{3}-1\right)\left(c_{12} c_{21}-c_{11} c_{22}\right)}{c_{13}\left(c_{22} c_{31}-c_{21} c_{32}\right)+c_{12}\left(c_{21} c_{33}-c_{23} c_{31}\right)+c_{11}\left(c_{23} c_{32}-c_{22} c_{33}\right)}\right) .
\end{aligned}
$$

By assumption, each of the coordinates of the positive coexistence fixed point $E^{*}$ are positive, hence it belongs to $\mathbb{R}_{+}^{3}$.

\section{Appendix 2. Computations related to the 3D Ricker model}

Let us consider $F: \mathbb{R}_{+}^{3} \rightarrow \mathbb{R}_{+}^{3}$ to be the three dimensional Ricker competition model given by the following map

$$
F\left(x_{1}, x_{2}, x_{3}\right)=\left(x_{1} e^{r_{1}-x_{1}-a_{12} x_{2}-a_{13} x_{3}}, x_{2} e^{r_{2}-a_{21} x_{1}-x_{2}-a_{23} x_{3}}, x_{3} e^{r_{3}-a_{31} x_{1}-a_{32} x_{2}-x_{3}}\right) .
$$

We restrict ourselves to the case $0<r_{i}<1$, for $i=1,2,3$. We shall also assume that $F$ has a positive fixed point and an interior fixed point in each coordinate plane. This automatically imposes certain conditions on the parameters of the model. In fact, a computation shows that $F$ has the origin, $E_{1}=\left(r_{1}, 0,0\right), E_{2}=\left(0, r_{2}, 0\right)$, and $E_{3}=\left(0,0, r_{3}\right)$ as fixed points. In addition, the following fixed points in the coordinate planes

$$
\begin{aligned}
& E_{12}=\left(\frac{r_{1}-a_{12} r_{2}}{1-a_{12} a_{21}}, \frac{r_{2}-a_{21} r_{1}}{1-a_{12} a_{21}}, 0\right), \\
& E_{13}=\left(\frac{r_{1}-a_{13} r_{3}}{1-a_{13} a_{31}}, 0, \frac{r_{3}-a_{31} r_{1}}{1-a_{13} a_{31}}\right), \\
& E_{23}=\left(0, \frac{r_{2}-a_{23} r_{3}}{1-a_{23} a_{32}}, \frac{r_{3}-a_{32} r_{2}}{1-a_{23} a_{32}}\right),
\end{aligned}
$$

and the interior fixed point $E^{*}$ has coordinates given by

$$
\begin{aligned}
E^{*}= & \left(\frac{a_{12}\left(r_{2}-a_{23} r_{3}\right)+a_{13}\left(r_{3}-a_{32} r_{2}\right)+r_{1}\left(a_{23} a_{32}-1\right)}{a_{12} a_{21}+a_{13} a_{31}+a_{23} a_{32}-a_{12} a_{23} a_{31}-a_{13} a_{21} a_{32}-1},\right. \\
& \frac{a_{21}\left(r_{1}-a_{13} r_{3}\right)+a_{23}\left(r_{3}-a_{31} r_{1}\right)+r_{2}\left(a_{13} a_{31}-1\right)}{a_{12} a_{21}+a_{13} a_{31}+a_{23} a_{32}-a_{12} a_{23} a_{31}-a_{13} a_{21} a_{32}-1}, \\
& \left.\frac{a_{31}\left(r_{1}-a_{12} r_{2}\right)+a_{32}\left(r_{2}-a_{21} r_{1}\right)+r_{3}\left(a_{12} a_{21}-1\right)}{a_{12} a_{21}+a_{13} a_{31}+a_{23} a_{32}-a_{12} a_{23} a_{31}-a_{13} a_{21} a_{32}-1}\right) .
\end{aligned}
$$

For simplicity, let $p_{i}$ be the numerator of the $i$ th coordinate of $E^{*}$ and $q$ the common denominator in each coordinate. Thus, we are assuming that our parameters satisfy for $i, j=1,2,3$ and $i \neq j$

$$
r_{i}-a_{i j} r_{j}>0, \quad 1-a_{i j} a_{j i}>0, \quad \text { and } p_{i} \cdot q>0 .
$$

In the analysis that follows, we can also assume without loss of generality that $x_{i}<1$ as the max value of $x_{i}$ is $e^{r_{i}-1}<1$. Next, a computation shows that

$$
J F:=J F\left(x_{1}, x_{2}, x_{3}\right)=\left(\begin{array}{ccc}
\left(1-x_{1}\right) e^{K_{1}} & -a_{12} x_{1} e^{K_{1}} & -a_{13} x_{1} e^{K_{1}} \\
-a_{21} x_{2} e^{K_{2}} & \left(1-x_{2}\right) e^{K_{2}} & -a_{23} x_{2} e^{K_{2}} \\
-a_{31} x_{3} e^{K_{3}} & -a_{32} x_{3} e^{K_{3}} & \left(1-x_{3}\right) e^{K_{3}}
\end{array}\right),
$$


where

$$
\begin{aligned}
& K_{1}=r_{1}-x_{1}-a_{12} x_{2}-a_{13} x_{3}, \\
& K_{2}=r_{2}-a_{21} x_{1}-x_{2}-a_{23} x_{3},
\end{aligned}
$$

and

$$
K_{3}=r_{3}-a_{31} x_{1}-a_{32} x_{2}-x_{3} .
$$

Computing its determinant we obtain $\operatorname{det} J F=e^{K_{1}+K_{2}+K_{3}} \Delta$, where

$$
\begin{aligned}
\Delta= & 1-\left(x_{1}+x_{2}+x_{3}\right)+\left(1-a_{12} a_{21}\right) x_{2} x_{1}+\left(1-a_{13} a_{31}\right) x_{3} x_{1}+\left(1-a_{23} a_{32}\right) x_{2} x_{3} \\
& -\left(1-a_{12} a_{21}-a_{13} a_{31}-a_{23} a_{32}+a_{12} a_{23} a_{31}+a_{13} a_{21} a_{32}\right) x_{1} x_{2} x_{3} .
\end{aligned}
$$

The set $L C_{-1}=\left\{\left(x_{1}, x_{2}, x_{3}\right) \in \mathbb{R}_{+}^{3}: \operatorname{det} J F=0\right\}$ can be found by setting $\Delta=0$ and isolating the expression for $x_{3}$. Formally, this yields

$$
x_{3}=\Phi(x, y)=\frac{P\left(x_{1}, x_{2}\right)}{Q\left(x_{1}, x_{2}\right)},
$$

where $P\left(x_{1}, x_{2}\right)=1-x_{1}-x_{2}+x_{1} x_{2}-a_{12} a_{21} x_{1} x_{2}$ and $Q\left(x_{1}, x_{2}\right)=P\left(x_{1}, x_{2}\right)+a_{13} a_{31} x_{1}\left(1-x_{2}\right)+$ $a_{23} a_{32} x_{2}\left(1-x_{1}\right)+a_{12} a_{23} a_{31} x_{1} x_{2}+a_{13} a_{32} a_{21} x_{1} x_{2}$. We are looking for the region bounded by the component of $L C_{-1}$ closest to the origin, denoted by $\mathcal{R}_{1}$. We have that have $(0,0,0) \in \mathcal{R}_{1}$ and

$$
\partial \mathcal{R}_{1}:=L C_{-1}^{1}=\left\{\left(x_{1}, x_{2}, x_{3}\right): x_{3}=\frac{P\left(x_{1}, x_{2}\right)}{Q\left(x_{1}, x_{2}\right)}, P\left(x_{1}, x_{2}\right)>0\right\} .
$$

Observe that $P\left(x_{1}, x_{2}\right)>0$ whenever $x_{2}<\frac{1-x_{1}}{1-\left(1-a_{12} a_{21}\right) x_{1}}$. Also, from the definition of $Q\left(x_{1}, x_{2}\right)$, we see that if $P\left(x_{1}, x_{2}\right)>0$, then $Q\left(x_{1}, x_{2}\right)>0$, so it suffices to require that $P\left(x_{1}, x_{2}\right)>0$ in the definition of $L C_{-1}^{1}$.

To find the normal at each point in $L C_{-1}^{1}$, we compute

$$
\begin{aligned}
& \frac{\partial \Phi}{\partial x_{1}}=-\frac{\left(a_{13}\left(1-x_{2}\right)+a_{12} a_{23} x_{2}\right)\left(a_{31}\left(1-x_{2}\right)+a_{21} a_{32} x_{2}\right)}{Q\left(x_{1}, x_{2}\right)^{2}}<0 \\
& \frac{\partial \Phi}{\partial x_{2}}=-\frac{\left(a_{23}\left(1-x_{1}\right)+a_{13} a_{21} x_{1}\right)\left(a_{32}\left(1-x_{1}\right)+a_{12} a_{31} x_{1}\right)}{Q\left(x_{1}, x_{2}\right)^{2}}<0
\end{aligned}
$$

Since $\eta=\left(-\partial_{x_{1}} \Phi,-\partial_{x_{2}} \Phi, 1\right)$, we see that $\eta>0$ at $L C_{-1}^{1}$.

Next, we check the concavity of $L C_{-1}^{1}$ at the restriction of $L C_{-1}^{1}$ to each plane perpendicular to the $x_{1}$ and $x_{2}$ axes. This requires computation of $\partial_{x_{1} x_{1}} \Phi$ and $\partial_{x_{2} x_{2}} \Phi$. Before we begin, we observe that as we fixed one of the variables $x_{1}$ or $x_{2}$, we can view $Q\left(x_{1}, x_{2}\right)$ as a linear function in $x_{2}$ or $x_{1}$, respectively. Indeed, we can verify that

$$
Q\left(x_{1}, x_{2}\right)=\alpha_{1}\left(x_{1}\right)-x_{2} \beta_{1}\left(x_{1}\right)=\alpha_{2}\left(x_{2}\right)-x_{1} \beta_{2}\left(x_{2}\right),
$$

where for $\{i, j\}=\{1,2\}$ we have

$$
\alpha_{i}(t)=1-\left(1+a_{i 3} a_{3 i}\right) t
$$

and

$$
\beta_{i}(t)=1-a_{j 3} a_{3 j}-\left(1+a_{12} a_{23} a_{31}+a_{13} a_{21} a_{32}-a_{12} a_{21}-a_{13} a_{31}-a_{23} a_{32}\right) t .
$$

Thus, computing the second derivatives of $\Phi$, we obtain

$$
\frac{\partial^{2} \Phi}{\partial^{2} x_{1}}=-\frac{2 \beta_{2}\left(x_{2}\right)\left(a_{13}\left(1-x_{2}\right)+a_{12} a_{23} x_{2}\right)\left(a_{31}\left(1-x_{2}\right)+a_{32} a_{21} x_{2}\right)}{Q(x, y)^{3}}
$$


and

$$
\frac{\partial^{2} \Phi}{\partial^{2} x_{2}}=-\frac{2 \beta_{1}\left(x_{1}\right)\left(a_{23}\left(1-x_{1}\right)+a_{21} a_{13} x_{1}\right)\left(a_{32}\left(1-x_{1}\right)+a_{31} a_{12} x_{1}\right)}{Q(x, y)^{3}} .
$$

We claim that there are some conditions under which $\beta_{i}(t)>0$ for $t \in[0,1]$. Indeed, but modifying the notation, we can obtain a convenient simplification. Let us denote $a_{i j} a_{j i}=A_{k}^{2}$, for $\{i, j, k\}=\{1,2,3\}$. Notice that the relation $0<a_{i j} a_{j i}<1$ implies $0<A_{k}<1$. Then for $\{i, j\}=\{1,2\}$, we have

$$
\begin{aligned}
\beta_{i}(t) & =-t a_{12} a_{23} a_{31}-t a_{13} a_{21} a_{32}+t a_{12} a_{21}+t a_{13} a_{31}+t a_{23} a_{32}-a_{j 3} a_{3 j}-t+1 \\
& =-t a_{12} a_{23} a_{31}-t \frac{A_{3}^{2} A_{2}^{2} A_{1}^{2}}{a_{12} a_{23} a_{31}}+t A_{3}^{2}+t A_{2}^{2}+t A_{1}^{2}-A_{i}^{2}-t+1 \\
& =\left(1-A_{i}\right)\left(2 A_{j} A_{3} t+(1-t)\left(A_{i}+1\right)\right)+t\left(A_{3}-A_{i}\right)^{2}-t \frac{\left(A_{3} A_{2} A_{1}-a_{12} a_{23} a_{31}\right)^{2}}{a_{12} a_{23} a_{31}} .
\end{aligned}
$$

Under condition $\left(*_{2}\right)$, that is, whenever $a_{12} a_{23} a_{31}=a_{13} a_{32} a_{21}$, we have $A_{3} A_{2} A_{1}=a_{12} a_{23} a_{31}$. This implies that $\beta_{i}(t)>0$ and hence for $\{i, j\}=\{1,2\}$, we have

$$
\frac{\partial^{2} \Phi}{\partial^{2} x_{i}}=-\frac{2 \beta_{j}\left(x_{j}\right)\left(a_{i 3}\left(1-x_{j}\right)+a_{i j} a_{j 3} x_{j}\right)\left(a_{3 i}\left(1-x_{j}\right)+a_{3 j} a_{j i} x_{j}\right)}{Q\left(x_{1}, x_{2}\right)^{3}}<0 .
$$

